\title{
International Stellarator/Heliotron Database Progress on High-Beta Confinement and Operational Boundaries
}

\author{
A Weller ${ }^{1}, \mathrm{~K}$ Y Watanabe ${ }^{2}$, S Sakakibara ${ }^{2}$, A Dinklage ${ }^{1}$, H Funaba $^{2}$, J Geiger ${ }^{1}$, J H Harris ${ }^{3}$,

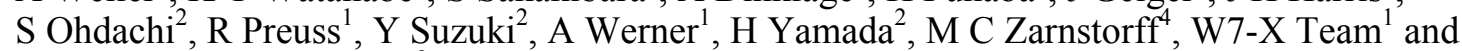 \\ LHD Experimental Group ${ }^{2}$ \\ ${ }^{1}$ Max-Planck-Institut für Plasmaphysik, IPP-EURATOM-Association, D-17491 Greifswald, Germany \\ ${ }^{2}$ National Institute for Fusion Science, 322-6 Oroshi-cho, Toki-City, Gifu 509-5292, Japan \\ ${ }^{3}$ Oak Ridge National Laboratory, Oak Ridge, TN 37831, USA \\ ${ }^{4}$ Princeton Plasma Physics Laboratory, Princeton, NJ 08543, USA \\ E-mail: arthur.weller@ipp.mpg.de
}

\begin{abstract}
The International Stellarator/Heliotron Confinement Database (ISHCDB) was extended by high- $\beta$ data compiled from the Large Helical System (LHD) and the W7-AS Stellarator. The main purpose is to enhance the basis for extrapolation of the global confinement properties to the reactor regime. The high- $\beta$ configurations and experimental achievements in both devices are briefly described. The impact of beta on the configuration parameters and the global confinement is discussed. In particular, the confinement data in the high- $\beta$ regime are compared with the ISS95 and ISS04 scaling laws which were derived from a database including relatively few high- $\beta$ cases. In addition, a Bayesian model comparison approach is used to test scaling predictions derived from basic confinement models. Unlike in tokamaks, the operational boundaries in stellarators and helical systems are determined by the available heating power and confinement properties rather than by disruptive stability or density limits. The role of a pressure induced equilibrium limit is discussed in particular. An attempt is made to compare the high- $\beta$ data with tokamak confinement and with operational boundaries observed in tokamaks. Further extensions of the database by parameters characterizing stability and local transport properties are proposed.
\end{abstract}

PACS numbers: $52.25 . \mathrm{Xz}, 52.30 . \mathrm{Cv}, 52.55 . \mathrm{Dy}, 52.55 . \mathrm{Hc}$

\section{Introduction}

The ultimate goal of the international stellarator program is to provide a basis for an economically attractive fusion energy source. The basic characteristic of stellarators and helical systems is the inherent capability of 'net-current free' operation. Actually, equilibrium currents and bootstrap currents exist and finite currents may be driven by the heating systems. However, the MHD equilibrium is primarily maintained by the external, necessarily non-axisymmetric fields. The prospects of the manifold ef different configurations and approaches have to be assessed by inter-machine comparisons of the achieved global plasma parameters and local energy and particle transport properties. Likewise, the collection of reference data from existing machines will enable evaluation of the benefit of configuration optimization as anticipated in the new Wendelstein W7-X stellarator [1] and in the National Compact Stellarator NCSX [2] being presently under construction ${ }^{5}$. Scaling expressions of the global confinement have been established in the frame of the International Stellarator/Heliotron Confinement Database (ISHCDB) activity [3]. The first inter-machine confinement scaling analysis was based on data obtained for different low- $\beta$ ECRH (electron cyclotron resonance heating) and NBI (neutral beam injection) scenarios in several stellarators and helical devices. Here, beta defined by

\footnotetext{
${ }^{5}$ The NCSX project was closed in 2008
} 
$\langle\beta\rangle=2 \mu_{0}\left\langle p / B^{2}\right\rangle$, is the volume averaged plasma pressure normalized to the magnetic field pressure.

The resulting ISS95 scaling law, widely used as a reference for characterizing global energy confinement in stellarators and helical systems, features gyro-Bohm type confinement and is described by a power law for plasma and engineering parameters in the following form:

$$
\tau_{E, I S S 95}=0.256 \cdot R^{0.65} \cdot a^{2.21} \cdot B^{0.83} \cdot \bar{n}^{0.51} \cdot P^{-0.59} \cdot t^{0.4}
$$

Here, $\tau_{E, I S S 95}[\mathrm{~s}]$ is the predicted energy confinement time, and the parameters are the major radius $R[\mathrm{~m}]$, the effective minor plasma radius $a[\mathrm{~m}]$, the volume averaged magnetic field $B[\mathrm{~T}]$, the volume averaged electron density $\bar{n}\left[10^{20} \mathrm{~m}^{-3}\right]$, the effective heating power $P[\mathrm{MW}]$, and the rotational transform $+($ at $\mathrm{r}=2 / 3 \mathrm{a})$, respectively.

A substantial extension of the parameter regime, mainly in LHD, has resulted in a corresponding enhancement of the ISHCDB database, which also included a first high- $\beta$ dataset from W7-AS. A new unified scaling of the confinement time in stellarators was proposed (ISS04,[4]) which basically confirmed the parameter dependencies of the ISS95 scaling and the gyro-Bohm dependence:

$$
\tau_{E, I S S 04}=0.465 \cdot R^{0.64} \cdot a^{2.28} \cdot B^{0.84} \cdot \bar{n}^{0.54} \cdot P^{-0.61} \cdot t^{0.41}
$$

In order to cope with offsets between different data subsets in the database, empirical renormalization factors, depending on the configuration or device, were introduced, so that general trends of the confinement in net-current free stellarator plasmas as well as individual configuration dependent properties can be described. Both, the ISS95 and ISS04 scaling expressions were achieved under the constraint of dimensionally correct fitting, so that the confinement scaling can be converted into a relation using important dimensionless physical parameters:

$$
\tau_{E, I S S 04} / \tau_{B o h m} \propto \rho^{*-0.79} \cdot \beta^{-0.19} \cdot v^{* 0.00} \cdot t^{1.06} \cdot \varepsilon^{-0.07}
$$

Here, the Bohm time $\tau_{\text {Bohm }}$ is defined by $a^{2}$ divided by the Bohm diffusion coefficient. The parameters $\rho^{*}, \beta$, and $v^{*}$ are the ion gyro-radius normalized to the plasma radius $a$, the kinetic plasma pressure normalized to the magnetic field pressure, and the ion collision frequency, normalized to the bounce frequency of toroidally trapped ions, respectively. The last parameter is the inverse aspect ratio $\varepsilon=a / R$. The rho-star dependence is close to gyro-Bohm scaling, similarly as in the case of ISS95. The scaling relations are consistent with only a weak dependence on beta and no dependence on nue-star. However, the database did not contain a sufficiently large subset of high- $\beta$ cases. Therefore, an effort is made to extend the high- $\beta$ data subset in the ISHCDB by data from W7-AS and LHD in order to clarify the role of the plasma beta and also of the collisionality.

A compilation of high- $\beta$ results in LHD and W7-AS including comparisons among each other as well as to tokamak results was reported [5-9]. Until 2006-2008 further significant progress has been achieved in LHD reaching volume averaged values of $\langle\beta\rangle \approx 5 \%$ [10-12]. These new data are particularly important to include in the ISHCDB / ISHPDB, since they bridge the gap to the reactor relevant $\beta$-values (here, ISHPDB is the acronym for International Stellarator/Heliotron Profile Database). Key issues concern the $\beta$-dependence of the confinement and the physical mechanisms determining the achievable stationary level of $\langle\beta\rangle$.

In order to get a more detailed understanding of the energy transport in stellarators and helical devices the international database effort is presently extended by the so-called International Stellarator/Heliotron Profile Database (ISHPDB,[13]). This activity aims at the documentation and analysis of 1-d and 2-d data for various topics including local energy and particle transport. With regard to high- $\beta$ physics, first studies of the effect of the magnetic configuration on the local transport in LHD high- $\beta$ discharges have been made [14]. In addition, the definition of an appropriate set of configuration and plasma parameters is required to characterize the dependence and impact of ideal and resistive MHD modes on the magnetic configuration [15]. 
In this paper, the main focus is to characterize and compare different sets of high- $\beta$ data from LHD and W7-AS and to discuss their implementation in the ISHCDB. Some preliminary results on the scaling of the global confinement will be presented. In particular, the W7-AS and LHD high- $\beta$ survey datasets are used to identify differences in the confinement compared to low- $\beta$ plasmas by a probabilistic model comparison approach [16,17]. Finally, operational limits are discussed with reference to tokamaks, and some remarks about possible extensions towards MHD related data will be made.

\section{W7-AS and LHD device characteristics}

Wendelstein W7-AS $[18,19,9]$ was a medium size stellarator $(R=2 \mathrm{~m}, a \leq 0.18 \mathrm{~m})$ operated until mid of 2002. The five-periodic magnetic field is partially optimized in respect of MHD properties (reduced Pfirsch-Schlüter currents) and neoclassical transport by three-dimensional (3D) shaping. The magnetic field $(\mathrm{B} \leq 2.5 \mathrm{~T})$ is generated by a system of 45 non-planar modular coils providing a rotational transform of $\boldsymbol{t}_{\text {vac }} \approx 0.4$. W7-AS is characterized by low shear allowing for avoiding low order iotaresonances, and a magnetic well over the entire plasma to ensure MHD stability (except at very low $\beta$ in the presence of a large inward shift). The vacuum rotational transform can be varied in the range $0.25 \leq \boldsymbol{t}_{\text {vac }} \leq 0.65$ by an extra set of 10 planar toroidal field coils. Additionally, poloidal field coils allow for adjustments of the horizontal plasma position. Current drive and current control is accomplished by an Ohmic transformer. Since 2000 W7-AS was equipped with a modular island divertor system [20] including a set of 10 in-vessel, so-called control coils for controlling the width of edge islands by resonant field perturbations $B_{5 m}$. High- $\beta$ plasmas were heated by almost tangential neutral beam injection with beam energies of $50-55 \mathrm{keV}$ yielding port-through heating powers of $P_{N B I} \leq 3.4 \mathrm{MW}$. The heating efficiency decreases towards lower fields restricting high- $\beta$ operation to $\mathrm{B} \geq 0.7 \mathrm{~T}$.

The Large Helical Device (LHD) [21] is the largest existing heliotron type helical device $(R=3.9 \mathrm{~m}$, $a \leq 0.65 \mathrm{~m})$. The magnetic field of 10 field periods $(\mathrm{B} \leq 3 \mathrm{~T})$ is produced by a pair of superconducting helical windings (heliotron type configuration). Three sets of poloidal field coils are used to change the axis preset position of the vacuum configuration in the range $R_{a x}=3.4-4.1 \mathrm{~m}$ and for plasma shaping. The profile of the vacuum rotational transform in LHD features much higher shear compared with W7-AS. Central and edge values are in the range $\boldsymbol{t}_{\text {vac }}(0) \geq 0.35$ and $\boldsymbol{t}_{\text {vac }}(a) \leq 1.5$, respectively. The inward shifted vacuum configuration $\left(R_{a x}=3.6 \mathrm{~m}\right)$ has no magnetic well, but it develops in the center and expands as $\beta$ is raised. The plasma edge remains in a magnetic hill region. The rotational transform, and at the same time the aspect ratio, can be varied by changing the center of the current in the three-layer helical coils. In addition, a set of external saddle coils allows to drive an $\mathrm{n} / \mathrm{m}=1 / 1$ magnetic island at the plasma edge, which can be utilized for local island divertor (LID) operation and island studies [22]. The heating power of $P_{N B I} \leq 14 \mathrm{MW}$ (port-through) is provided by 3 tangential beam-lines using negative ions with beam energies of $150-180 \mathrm{keV}$. In addition, a fourth $40 \mathrm{keV}$, positive ion based, radial injector can deliver a port-through power of 6-7 MW. However, the present data compilation only includes cases with negative ion based NBI.

\section{High Beta Datasets, Configuration Effects}

The use of the database for inter-machine comparisons depends crucially on a clear definition of a set of key parameters and on standardized analysis procedures. However, in the high- $\beta$ regime the configuration changes with $\beta$, and hence some parameters are not easily accessible, and their definition has to be reconsidered. Most importantly, the identification of the plasma boundary and hence the determination of the effective plasma radius requires a more sophisticated analysis, since the plasma edge region of high- $\beta$ plasmas is usually characterized by stochastic field layers where a significant pressure gradient is still maintained [23-25]. For practical reasons, the measured pressure profiles are mostly fitted by equilibria based on the assumption of nested flux surfaces as calculated with the VMEC code [26]. In LHD, the flux contour which contains $99 \%$ of the measured kinetic plasma energy has turned out to be the most appropriate measure of the plasma edge location. 


\subsection{W7-AS High Beta Data}

In W7-AS, high- $\beta$ discharges are effectively limited by the divertor structures, and therefore the plasma boundary is identified by the intersection of flux surfaces with the divertor. The determination of the proper effective plasma radius is achieved by using the STELLOPT code [27] which is based on VMEC and iterates for equilibria consistent with the measured diamagnetic energy and kinetic data [28]. The pressure induced shift of the plasma axis can be compensated by an appropriate vertical field so that plasmas with the maximal possible plasma volume could usually be established.

A first database consisting of about 200 entries was compiled in 2003 [6] based on cases for which dedicated VMEC or STELLOPT calculations were made for different reasons. First comparisons of these data with those of LHD were published in 2006 [7]. Standard parabolic pressure profiles were used in the VMEC calculations and (small) net-currents were modeled with a standard current profile. In individual cases it could be shown that the experimental pressure profiles were close to parabolic apart near the plasma edge. The equilibria were calculated in such a way as to reproduce the measured diamagnetic energy and the plasma boundary just in contact with the plasma facing structures (divertor troughs). Most of the configuration parameters (effective plasma radius $a$, axis position, values of the rotational transform, volume averaged magnetic field, etc. as well as plasma data such as $\langle\beta\rangle$ were taken from the VMEC calculation.

A second compilation was made in 2006 , which was closely dedicated to a refined analysis of the high- $\beta$ confinement in W7-AS by a probabilistic model comparison approach (see section 4.4 and references therein). Here, a systematic W7-AS database search was made that was constrained to find all high- $\beta$ cases without significant toroidal plasma current. In a second step, only discharges were selected, which showed a quasi-stationary behaviour (for $\Delta t \gg \tau_{E}$ ). Using one or more time points per discharge during quasi-stationary periods almost 400 entries in this database were generated. The majority of these entries (about 310) was newly-created. Only about 90 out of 220 entries of the first 2003 database were selected for inclusion in this more recent compilation. Although, there is a good agreement between the two datasets, the 2006 database is to be preferred because of the more comprehensive and more stringent data selection. The data cover a variety of different configurations with the majority of cases close to the optimum confinement at $t \approx 1 / 2$ (rotational transform, iota). The achieved $\beta$-values in W7-AS, taking the 2006 dataset, are shown in figure 1, plotted versus a time characterizing the duration of the quasi-stationary period around the $\beta$-maximum (normalized to the confinement time). The dashed horizontal lines indicate rough estimates of equilibrium limits based on a simple model for a critical Shafranov shift $(\Delta=0.5 a)$ for two particular configurations spanning the range in the rotational transform. The Shafranov shift is considered to provide a good index for the deformation of magnetic flux surfaces and for characterizing the proximity to an equilibrium limit (see section 5.1).

\subsection{LHD High Beta Data in inward shifted configurations $\left(R_{a x}=3.6 \mathrm{~m}\right)$}

The first comprehensive survey of high- $\beta$ data covering the experimental period of fiscal years 2004 and 2005 made use of a similar constrained database search [7]. During the present study this survey data compilation was revised substantially. Firstly, the data search was extended to include new data from fiscal years 2006 and 2007. Secondly, three particular configurations were selected, and the data were grouped accordingly, in order to facilitate studies of configuration effects on the high- $\beta$ confinement. Thirdly, entries connected with transient phases (e.g. pellet-injection and switching of NBI power), with operation times of the radial neutral beam injector (for which the heating efficiency was not well known), or with discharges of large non-hydrogen admixtures were rejected. Fourthly, the assessment of the absorbed heating power, which is a particular issue in the high- $\beta$ regime, was improved by increasing the database of pre-calculated NBI heating efficiencies. Finally, the determination of equilibrium parameters from the VMEC database was modified resulting in an improved consistency with measured pressure profiles. Thus, three "survey" datasets with altogether about 806 selected entries referring to configurations with a vacuum axis position of $R_{a x}=3.6 \mathrm{~m}$ and helical coil (HC) pitch parameters of $\gamma=1.25$ (standard, 91 entries), 1.22 (186 entries) and 1.20 (optimum, 529 entries) were compiled at the maximum of the diamagnetic energy within each discharge. Here, the pitch parameter is defined by 
$\gamma=M / L / A_{c}$, where $L=2, M=10, A_{c}=3.6-4.4$ (number, periods and aspect ratio of $\mathrm{HCs}$ ). The aspect ratio of the $\mathrm{HC}$ is varied by changing the current ratio in the three $\mathrm{HC}$ layers resulting in plasma aspect ratios of $A_{p}=5.7,6.1$ and 6.5. These configuration parameters refer to the vacuum configurations. Since the rotational transform in LHD scales as $A_{p} \sim t$, the Shafranov shift is $\Delta / a \sim 1 / A_{p}$, and hence is progressively reduced in configurations with decreasing $\gamma$ parameter. This has proven to be a key to maximize the achievable beta.

The $\langle\beta\rangle$ values achieved in these three configurations are shown in figure 2 in a similar form as the W7-AS data in figure 1 using the survey data described above. Parameters of the vacuum configurations (plasma volume, volume averaged magnetic field) and diamagnetic measurements of the plasma energy were used in the evaluation taken from the LHD database. The dependence of the maximum achievable $\beta$ values on the aspect ratio or alternatively on the rotational transform shows a correlation with $\langle\beta\rangle_{\text {crit }} \approx t^{2} / A_{p}$ (horizontal dashed lines) which defines an equilibrium limit for a classical low-shear stellarator (in a low- $\beta$ approximation) and which is taken as a reference. Here, the central rotational transform is used. Therefore, the actual limits for LHD are significantly larger because of the large positive shear in the $t$-profile. A more detailed discussion of the possible role of an equilibrium limit is made in section 5.1. Actually, the observed Shafranov shift stays clearly below the critical value (half of the minor radius), even at the maximum beta of $5 \%$, where $\Delta / a \approx 0.33$. It should be noted that for W7-AS $\langle\beta\rangle_{\text {crit }} \approx 2 t^{2} / A_{p}$ was taken to account for the reduction of the Shafranov shift by a factor of about two predicted for the partially optimized stellarator configuration in W7-AS [6]. In addition to its direct connection with an equilibrium beta limit, a large Shafranov shift also leads to unfavourable NBI power deposition in LHD and subsequent large direct fast ion losses at low magnetic fields as calculated by the FIT code [29]. Depending on the configuration, this results in different operational limits at low magnetic fields and differences in the achievable beta, and may therefore provide an alternate explanation for the dependence on the $\gamma$-parameter as shown in figure 2 . On the other hand, any configuration dependent MHD stability effects can be excluded, since the observed magnetic fluctuation levels, mostly due to $(m, n)=(3,2)$ modes located in the edge region, are largest in the optimized configurations with higher $\beta$-limits.

In addition to the described survey datasets, four more high- $\beta$ datasets of configurations with a vacuum axis position of $R_{a x}=3.6 \mathrm{~m}$ were compiled and inserted or prepared for inclusion, respectively, in the ISHCDB. These data were mainly dedicated to studies of the effect of the magnetic configuration and the plasma $\beta$ on the confinement, in particular by local transport analyses [30,14]. Therefore, these data are dubbed "transport data" in this work in order to distinguish them from the survey data. A different approach was used for the analysis and compilation of these data. In particular, a more detailed assessment of the equilibrium parameters was made for each individual case by using measured kinetic data (electron pressure profiles from Thomson scattering), which were fitted with pre-calculated VMEC equilibria with the boundary shape calculated by the HINT code [25]. Also, the absorbed NBI heating power was calculated for each case, and a careful selection of quasi-stationary time intervals in the high- $\beta$ discharges was made. In the case of the standard configuration $(\gamma=1.25), \sim 230$ entries were collected, for the $\gamma=1.22$ case two datasets with $\sim 220$ and 108 entries, and for the $\gamma=1.20$ configuration $\sim 340$ entries, respectively.

\subsection{New regime of high central beta in $L H D$}

A new operation regime of high central beta plasma was found in the Large Helical Device [12]. Peaked pressure profiles could be established which are formed in the recovery phase of the electron temperature after sequentially injected hydrogen pellets. A key parameter is the (preset) location of the vacuum magnetic axis which has to be shifted outward in order to produce a super-dense core (SDC) with an internal diffusion barrier (IDB) induced by pellet injection [31,32]. While the electron density decreases after pellet injection, the electron temperature recovers quickly. In this recovery phase, peaked pressure profiles are observed. In outward shifted configurations not only is a larger density increase obtained by pellet injection, but also very importantly, the MHD stability is improved in the 
plasma core due to the existence of a magnetic well so that steeper pressure profiles can be maintained. In turn, the pronounced peaking of the pressure profiles leads to further improvement of the core stability by the formation of a deeper magnetic well induced by a larger Shafranov shift of the plasma. As shown in figure 3, central beta values as large as in the case of the conventional inward shifted high-beta plasma (\#69910 in figure 3) can be achieved by this alternative approach. The pressure profiles (\#80586, \#69286 in figure 3) are clearly more peaked, where the peaking factor is given by the ratio between the central and the volume averaged plasma beta. Due to the resulting comparably low volume averaged $\beta$, the relatively high magnetic fields and the outward shift of the IDB cases they were excluded in the present database approach. The profiles of the plasma parameters and a set of parameters characterizing the observed MHD activities in this high central beta operation, however, will be included in the planned MHD database (see section 6).

\section{Global confinement}

\section{1. $W 7-A S$}

The experimental and numerical assessment of the absorbed NBI heating power is subject to relatively large errors due to the increased fraction of injected NBI ions suffering direct orbit losses at low magnetic field (the high- $\beta$ data were obtained with toroidal magnetic fields in the range $B_{t}=0.75 \mathrm{~T} \ldots 1.25 \mathrm{~T}$ ). In figure 4 , a compilation of the high- $\beta$ energy confinement times normalized to the values predicted by the ISS95 and ISS04 scaling relations [3,4] is given as a function of $\langle\beta\rangle$. Although a detailed equilibrium analysis with the PIES code [23] revealed a deterioration of the local transport in the outer plasma region with increasing beta due to the expansion of the stochastic layer (see section 5), no direct evidence of a degradation of the confinement with increasing $\beta$ is found in the dataset. On the contrary, the data suggest some confinement improvement towards high $\beta$, which might be connected with a deepening of the magnetic well and the respective improvement of the MHD stability [7]. However, in the majority of cases the configuration was optimized for achieving high $\beta$, whereas the data in the lower $\beta$-range were partially taken under unfavourable conditions. An indication for this could be the low value of the $\mathrm{H}$-factor at low $\beta$ where $H_{I S S O 4}<1$. Therefore, a clear conclusion about the dependence of the confinement on $\beta$ cannot be given at present.

\section{2. $L H D$}

In the three survey datasets the vacuum boundary was used for the evaluation of the confinement scaling laws. The vacuum boundary is close to the contour encompassing $99 \%$ of the total pressure at high beta which is considered to be a proper index for the finite- $\beta$ boundary [25], and which was used in the analysis of the transport data. In contrast, the ideal finite- $\beta$ equilibrium calculations give lower values for the plasma radius. Here, some discrepancy between HINT and VMEC as regards the magnitude of the Shafranov shift has to be stated which is not yet fully understood. Although the HINT results are in better agreement with the experimental data, VMEC data are still widely used because much more pre-calculated equilibria are available in the LHD equilibrium database. The values of the rotational transform and of the plasma position required for the global scaling law predictions have been derived using interpolations between pre-calculated VMEC equilibria which were selected to reproduce the available experimental data related to the flux surface geometry.

In figure 5 , high- $\beta$ energy confinement times normalized to the values predicted by the ISS 95 and ISS0 4 scaling relations are plotted in a similar way as for W7-AS in figure 4. Here, the survey data are compared with the transport data, which have been compiled for detailed confinement and MHD studies during stationary time intervals and using a comprehensive analysis of plasma and configuration parameters (see section 3.2). Due to technical reasons, the transport data for the $\gamma=1.22$ and 1.25 cases are arranged each in two datasets. The analysis approach, however, is equivalent for the two datasets marked by "transport" and "transp(2)" in the figure legends. The progressive degradation of the confinement towards high beta has been described earlier [33] and attributed to a large extent to the beta-induced changes of the flux geometry parameters (outward shift of the configuration). Actually this is confirmed by the lower part of figure 5 where ISS04 renormalization factors are used for taking the effect of different axis positions into account. Here, the original ISS04 renormalization 
factors, depending on the axis position of the vacuum configuration were interpolated at the position of the finite- $\beta$ configurations represented by the center of the flux surface with $r / a=2 / 3$. Only in the case of one of the transport datasets $(\operatorname{transp}(2))$ the center position of the flux surface with $r / a=2 / 3$ was not available in the data file, and therefore, the axis position was taken instead. After the renormalization, almost no dependence of the $\mathrm{H}$-factor on the plasma beta is left. Detailed local transport investigations, however, have revealed an increase of the effective heat conduction coefficient close to the plasma periphery [14], which cannot be attributed to the shift of the configuration but rather to increased g-mode turbulence under the influence of the magnetic hill or stochasticity [30].

\subsection{Comparison with tokamak confinement}

The confinement in tokamaks depends crucially on the toroidal current, which enters into the tokamak scaling expressions based on a power law of the engineering parameters. The IBP98 $(y, 2)$ ELMy H-mode global confinement expression [34], given by

$$
\tau_{E, I P B 98(y, 2)}=0.144 \cdot R^{1.39} \cdot a_{h}^{0.58} \cdot B^{0.15} \cdot I^{0.93} \cdot \bar{n}^{0.41} \cdot M^{0.19} \cdot P^{-0.69} \cdot \kappa^{0.78}
$$

was used for comparison with the W7-AS and LHD high- $\beta$ survey data. Here, the same units are used as in equations (1) and (2). Additional parameters are the toroidal plasma current $I[\mathrm{MA}]$, the average ion mass $M$ [amu], and the plasma elongation $\kappa$. The minor radius $a_{h}$ is the horizontal plasma radius which is related to the effective plasma radius $a$ by $a_{h}=a / \sqrt{\kappa}$. In order to perform the comparison, we make the assumption, that the stellarator rotational transform can be replaced by that of an equivalent tokamak current which generates the same rotational transform. The relation between the rotational transform and the current can be expressed by [35,36]:

$$
I_{e q}=f(\varepsilon) t(2 / 3 a)\left(5 a^{2} B / R\right)\left[1+\kappa^{2}\left(1+2 \delta^{2}-1.2 \delta^{3}\right)\right] / 2 \kappa
$$

Here, the rotational transform at $r / a=2 / 3$ is chosen as a representative value for the confinement region [3]. The geometry factor $f$ depends on the inverse aspect ratio $\varepsilon=a_{h} / R$ and is given by $f(\varepsilon)=(1.17-0.65 \varepsilon) /\left(1-\varepsilon^{2}\right)^{2}$. In addition to the mean plasma elongation $\kappa$, the mean triangularity is included in (5), represented by the parameter $\delta$. The mean plasma shape parameters in W7-AS and in LHD are $\kappa \approx 2, \delta \approx 0.3$ and $\kappa \approx 1, \delta \approx 0$, respectively. The result of the comparison, shown in figure 6 , suggests that the high- $\beta$ confinement in W7-AS and LHD is quite comparable with ELMy H-mode confinement in tokamaks. Actually, if the IBP98 $(y, 2)$ scaling (equation (4)) is transformed into an expression using dimensionless physics parameters [34,4], a much stronger $\beta$-, $t$ - and $\varepsilon$-dependence appears compared with ISS04 (equation (3)):

$$
\tau_{E, I P B 98(y, 2)} / \tau_{B o h m} \propto \rho^{*-0.70} \cdot \beta^{-0.90} \cdot v^{*-0.01} \cdot M^{0.96} \cdot t^{3.0} \cdot \varepsilon^{0.73} \cdot \kappa^{2.3}
$$

\subsection{Connor-Taylor model comparison}

Scalings in which the relations between the parameters are consistent with basic physics laws and which have a dimensionally correct form are preferable for getting more insight in the confinement physics. In the case of a power law expression, this means, that constraints are imposed on the exponents of the parameters. In this work, scaling expressions constrained by six different basic so-called Connor-Taylor (CT) models [37,38] related to confinement physics were tested. The models originate from the assumption that the scaling relation for the energy content should show the same invariances under similarity transformations as a distinct set of equations regarded essential for the description of basic plasma behavior. E.g., to describe a collisionless plasma the Fokker-Planck equation without a collisional term will be of importance, where in order to describe beta effects the Maxwell equations come into play. For this purpose, Bayesian probability theory for model comparison was used and applied to subsets of low- $\beta$ (W7-AS) and high- $\beta$ (W7-AS, LHD) global confinement data $[16,17]$ to determine the model which provides the best fit to the experimental data. Imposing the invariance constraint above leads to a generalized power law ansatz for the plasma energy of the form 


$$
W^{\text {theo }}=n a^{4} B^{2} \sum_{k} c_{k}\left(\frac{P}{n a^{4} B^{3}}\right)^{\xi_{1 k}}\left(\frac{a^{3} B^{4}}{n}\right)^{\xi_{2 k}}\left(\frac{1}{n a^{2}}\right)^{\xi_{3 k}} .
$$

The approach also allows a refined analysis of the global confinement including the description of saturation effects in the control parameters such as the averaged density $n$ or the absorbed heating power $P$. The remaining parameters are the magnetic field strength $B$ and the effective plasma radius $a$.

According to the invariance principle [38] the exponents $\xi$ in this generalized scaling relation (equation (7)) are subject to constraints depending on the used model. Models that ignore any finite- $\beta$ effects were found to fit the low- $\beta$ data best, whereas finite- $\beta$ models gave the best agreement with the high- $\beta$ data. Therefore, it is concluded that the global confinement depends on beta in this range. The present results for the high- $\beta$ survey data are summarized in table 1 . In all data subsets high- $\beta$ models appear to fit the data best, as can be expected. Since the W7-AS high- $\beta$ data are in a different collisionality region well separated from the LHD data (figure 7), the role of the collisionality may be assessed as well. A clear dependence of the confinement time (normalized to ISS95) on the collisionality parameter was found in the high collisionality regime in LHD [39]. Moreover, this survey indicates that LHD data even allow for a detailed assessment of the transition from low- to high- $\beta$ regimes. Whereas in W7-AS the collisional high- $\beta$ model is favoured, the model comparison clearly identifies the collisionless high- $\beta$ model to be most probable in the case of the LHD high- $\beta \quad \gamma=1.20$ survey data (table 1). To check this result and to exclude effects of some co-linearity between the plasma $\beta$ and collisionality of the data (see figure 7), the analysis was performed on $\beta<3.5 \%$ and $v^{*}>1$ subsets of the complete dataset as well. However, no change of the major result was obtained, in all these subsets the collisionless high- $\beta$ type persisted within the CT approach. A preliminary analysis for the survey data of the LHD configurations with $\gamma=1.25$ and $\gamma=1.22$ gave inconclusive results with regard to the collisionality which are ascribed to a much lower number of available data.

The Bayesian approach provides a confidence range where predictions and extrapolations can be trusted given the experimental errors. An example for the prediction of a single parameter dependence (here: density dependence) and comparison with a dedicated experimental parameter scan is shown in figure 8 . The multi-parameter dependence according to equation (7) was derived from all data of the W7-AS high- $\beta$ subset (open symbols) which are given here in terms of plasma energy as a function of plasma density. The solid line represents the resulting dependency on the density alone (all other control parameters kept constant) together with its confidence range (shadowed). The predicted curve agrees very well with the data of a dedicated density scan (solid symbols) which were not included in the original dataset.

\section{Operational Boundaries}

\subsection{Equilibrium limit}

In both experiments, LHD and W7-AS, the $\beta$-limit is not determined by a disruptive response to MHD instabilities. More likely, a deterioration of the equilibrium has to be considered. Pfirsch-Schlüter (PS) currents can cause a significant Shafranov shift in high- $\beta$ configurations. The Shafranov shift is considered to provide a good index for the deformation of magnetic flux surfaces. In a circular geometry, the classical equilibrium limit corresponds to a Shafranov shift reaching $\Delta / a \approx 1 / 2$. In the tokamak case, the inboard poloidal field due to the toroidal plasma current is cancelled by the vertical field used for maintaining plasma equilibrium. Hence, a separatrix appears in the plasma with detrimental influence on the confinement. In stellarators, the Pfirsch-Schlüter currents drive resonant field perturbations which can lead to ergodic equilibrium fields. This is associated with enhanced transport, and therefore, magnetic field stochastization may eventually be a major cause of an equilibrium $\beta$-limit in non-axisymmetric devices. The minimization of the Shafranov shift by 3D flux surface shaping has been one of the most important design criteria for the W7-X configuration. This optimization is required not only because of the equilibrium limit issue, but also because the edge 
topology should not depend too much on $\beta$ in order to allow stable divertor operation. The present high- $\beta$ database provides an important reference to verify the benefit of configuration optimization.

\subsubsection{Analytic predictions of the Shafranov shift}

In a linearized low- $\beta$, and low-shear approximation the Shafranov shift for a classical stellarator can be given by $\Delta / a \approx A_{p}\langle\beta\rangle / 2 t^{2}$ [40]. Using the condition $\Delta / a \approx 1 / 2$, estimates of the critical $\beta$-values can then be obtained, which are indicated in figures 1 and 2 (where a factor of 2 was applied for W7AS to account for the partial configuration optimization, and the central iota-value was used in LHD). Detailed analytical work elucidating the role of equilibrium and stability limits in stellarators can be found in review articles by Shafranov [41] and Pustovitov [42]. Based on the work of Miyamoto, the analytic formulation for a plasma with an on average circular cross section was extended to drop the low- $\beta$ constraint. The new axis position is determined by an equation based on the toroidal vector potential $A_{\varphi}$ :

$$
0=\left(\frac{\partial A_{\varphi}}{\partial R}\right)_{\theta=0}=\left(\frac{B_{0}}{R_{0}} t(r) r-\frac{1}{2}\left(\frac{A_{P S}(a)}{a}+A_{P S}^{\prime}(a)\right)+A_{P S}^{\prime}(r)+H\right)_{r=\Delta}
$$

where $A_{P S}(r)$ is the part of the vector potential arising from the PS-currents and $B, R$ are the main field strength and the major radius, respectively. $H=\frac{1}{2}\left(A_{P S}^{\prime}(a)-A_{P S}(a) / a\right)$ arises from a $\beta$ dependent vertical field component if a fixed boundary constraint is applied, thus removing the part of the axis-shift which results by the shift of the plasma as a whole. Setting $H(r)$ to zero leads to the equation for the free-boundary case. The functional dependency of $A_{P S}(r)$ is given by the inhomogenous solution of the differential equation for $A_{\varphi}$ (see Miyamoto) with the toroidal current density as inhomogeneity. The relations are given by

$$
-\mu_{0} \hat{j}_{\varphi}=-\frac{2 \mu_{0} p^{\prime}(r)}{B t(r)} \approx \sum_{n=1}^{N} g_{n} r^{n}, \quad A_{P S}(r)=\sum_{n=1}^{N} \frac{g_{n}}{(n+2)^{2}-1} r^{n+2}
$$

with $g_{n}$ being the coefficients of the polynomial expansion for the radial dependency of the PScurrents without the cosine dependence $\left(\hat{j}_{\varphi}\right)$. For the classical $1=2$ stellarator (constant iota-profile) and a parabolic pressure profile $\left(p^{\prime} \propto-r\right)$, a quadratic formula results for $\Delta$, of which the relevant solution is given by

$$
\frac{\Delta}{a}=\frac{1}{G}\left(\sqrt{1+\frac{1}{3} G^{2}}-1\right), \quad \text { with } G=\frac{3}{2} \frac{R_{0} \beta_{0}}{a t^{2}} .
$$

The general square root dependence turns into a linear dependence of the Shafranov-shift on $\beta$ by expanding the square root in the limit $G^{2}<<3$. The latter condition corresponds to small $\beta$-values since aspect ratio and iota are fixed. Considering the free-boundary case changes the factor in front of $G^{2}$ by a factor of 2 , i.e. $1 / 3$ to $2 / 3$. The full implicit equation for the Shafranov-shift can also treat the case with shear, but it has then to be solved numerically. This has been done for LHD-like parameters and is shown in figure 9. The main effect of a rotational transform profile increasing towards the plasma edge is the reduction of the "integral" term $\frac{A_{P S}(a)}{a}$ reflecting the smaller PS-currents caused by the higher iota-values towards the boundary. Thus, the axis-shift is reduced by about a factor of two compared to the shearless case. This is roughly in agreement with the experimental findings and the much more accurate simulations using 3D equilibrium codes like HINT [43] or HINT2 [24]. Also the difference in the Shafranov shift predicted for the LHD cases becomes smaller in the presence of large shear. The relative large difference in the achieved beta in LHD shown in figure 2 may partially be caused by different experimental conditions. In particular, the NBI power was lower on average and the magnetic field higher in the standard configuration with $\gamma=1.25$. However, it has to be concluded again that equilibrium limit effects can hardly explain the LHD results. 


\subsubsection{Detailed equilibrium calculations for $W 7-A S$ and $L H D$}

Modelling of high- $\beta$ equilibria with the 3D MHD equilibrium code PIES [44] for a few selected W7AS cases required a modification of the original code to retain the experimentally observed pressure profiles. Figure 10 shows an example of a Poincaré plot corresponding to a PIES equilibrium of a $\langle\beta\rangle=1.9 \%$ case in W7-AS. It has to be noted that the plasma cross section in W7-AS varies between triangular and horizontally elongated shapes within each of the five toroidal field periods. The outermost contour in the Poincare plot indicates the boundary as determined by a VMEC/STELLOPT calculation which served as a start configuration for PIES. Similarly as found in LHD (see below), pressure and pressure gradients exist in the stochastic region (with embedded remnants of magnetic island chains) up to the VMEC boundary which is in contact with limiting structures. The degree of stochasticity, which increases with $\beta$, can be mitigated to some extent by using the control coils which are normally used for island divertor optimization. Actually, this has proven to be a key element for achieving $\langle\beta\rangle>3 \%$ in W7-AS. Radial transport in the stochastic region appears to be enhanced due to parallel transport associated with field line diffusion, limiting access to higher $\beta$. Such stochastization effects seem eventually to cause an equilibrium limit beyond the experimental data range in the optimum range of the rotational transform $(t \approx 1 / 2)$, where a separatrix and edge islands are usually present in the vacuum configuration. At lower iota, however, the content of resonant field harmonics in the vacuum configuration is lower. In this case, the achievable beta is clearly limited by an excessive Shafranov shift which is proportional to $t^{-2}[7,9]$.

Detailed equilibrium investigations in LHD with the HINT2 code [24] show field line stochastization effects in the plasma boundary region depending on the magnetic configuration and on $\beta$. Typically, the measured pressure profiles extend across the stochastic edge region up to or even beyond the boundary of the vacuum configuration. This is attributed to the relatively large connection lengths of the stochastic field lines compared with the electron mean free path lengths in the high collisional edge region. In figure 11, a HINT equilibrium for a $\langle\beta\rangle \sim 3 \%$ case is given, corresponding to the maximum beta achieved experimentally in the $\gamma=1.254\left(A_{p}=5.7\right)$ standard configuration. The Shafranov shift and the width of the stochastic layer seem to be too small to account for the limitation of $\beta$ due to the destruction of equilibrium surfaces. However, the impact of the quality of finite- $\beta$ flux surfaces on the transport has to be investigated further in more detail. Modelling studies of field line diffusion effects with the HINT2 code are underway to assess these effects quantitatively. In the case shown in figure 11 as well as in cases with up to the maximum beta of $5 \%$ in the optimized configuration $\left(\gamma=1.20, A_{p}=6.5\right)$, the observed Shafranov shift stays below $\Delta / a \approx 0.33$, and hence clearly below the critical value (half of the minor radius). The equilibrium limit in LHD seems to be not reached yet. Recent HINT2 results [45] suggest that this limit might be determined by the flattening of the pressure profiles due to stochastization rather than by an excessive Shafranov shift. Nevertheless, at maximum $\beta$, it appears that the confinement deteriorates when the Shafranov shift reaches some critical value. The Shafranov shift is associated with flux compression, which leads to a steepening of surface averaged profile gradients and hence increased transport fluxes [46]. Therefore, this effect of the flux surface geometry could contribute to the limitation of $\langle\beta\rangle$ without invoking flux surface destruction.

\subsection{Confinement limit and density limit}

Apart from the W7-AS low-iota configurations, where equilibrium limit effects are found, the maximum achieved beta in W7-AS and LHD is mainly determined by the available heating power. This can already be concluded from the discussion on the global confinement in section 4. Since the global confinement exhibits a favourable density scaling, high- $\beta$ plasmas are typically close to the density limit (see below). In figure 12 we discuss, whether the experimentally observed maximal $\beta$-values are consistent with the predictions of the global confinement scaling, or whether there is a limitation below this "confinement limit". The maximum $\langle\beta\rangle$ which can be expected for a given heating power was estimated on the basis of the ISS95 confinement scaling and taking the Sudo limit as the maximum for the achievable density. The experimental $\beta$-values (vertical axis) are close to their respective 
confinement limit values, plotted on the horizontal axis in figure 12. Actually, in the case of W7-AS (upper part) the experimental values exceed the confinement limit prediction because the densities are usually larger than the Sudo-densities (see figure 13 and discussion below) and $H_{\text {ISS95 }}>1$. The LHD data, shown in the lower part of the figure, refer to the $\gamma=1.22$ configuration, for which all high- $\beta$ data subsets are plotted. Here, some saturation effect is seen, which is consistent with the deterioration of the global confinement with increasing beta, as discussed in figure 5 (section 4.2). Hence the analysis suggests that the limits of $\langle\beta\rangle$ are roughly consistent with the transport underlying the ISS95 confinement scaling.

Typically, the averaged densities of the LHD high- $\beta$ datasets reach the Sudo-limit [47], particularly in the upper range of $\beta$. The Sudo density limit is given by

$$
n_{\text {Sudo-DL }} \equiv 1.11 \cdot(P \cdot B / V)^{0.5} \quad\left[10^{20} \mathrm{~m}^{-3}\right] \text {. }
$$

In W7-AS, the Sudo-limit is typically exceeded to some extent. The density limit scaling found in W7-AS $[48,49]$ is given in a slightly different form (for cases with dominant edge radiation) by

$$
n_{W 7-D L}=1.46(P / V)^{0.48} \cdot B^{0.54} \quad\left[10^{20} \mathrm{~m}^{-3}\right] \text {. }
$$

In equations (9) and (10), $V\left[\mathrm{~m}^{3}\right]$ is the plasma volume, and the heating power $P$ and magnetic field $B$ were already defined in connection with equation (1). The density limit in stellarators is not disruptive but defines the transition to deteriorated confinement at the beginning of a radiative collapse. This rather soft limit is not an absolute limit but depends on the available heating power. Figure 13 summarizes the comparison of different high- $\beta$ datasets with the Sudo-limit.

\subsection{Comparison with tokamak operational limits}

In order to characterize and compare the density range of stellarators / heliotrons and tokamaks a Hugill diagram representation is shown in figure 14. Here, $1 / q_{c y l}$ (where $q_{c y l}$ is the cylindrical equivalent safety factor), which is a measure of the tokamak current and plotted against normalized densities, was replaced by the value of the stellarator rotational transform at $r / a=2 / 3$. In addition to the data points of the W7-AS and LHD high- $\beta$ survey datasets, lines are drawn, which mark estimates of the Greenwald density limit for W7-AS and LHD. The Greenwald limit [50], given by $n_{G W L}=I_{e q} / \pi a^{2}$, is an absolute limit in tokamaks depending on the plasma current. Here, we adopt the same concept as introduced in section 4.3. The plasma current is replaced again by an equivalent current reproducing the rotational transform at $r / a=2 / 3$. The Greenwald boundary is different for W7AS and LHD because of the different geometry factors in equation (5), which was used to convert the rotational transform into an equivalent current. Most of the data are beyond the tokamak operational range which is bounded by $q_{c y l}=2$ (current disruption limit) and the Greenwald limit.

The operational diagram given in figure 15 combines both the tokamak Greenwald density limit and the tokamak stability limit due to ideal ballooning and free boundary kink modes, represented by the Troyon limit of $\beta_{N} \approx 3-4$. Here, the normalized beta is defined by $\beta_{N}=\langle\beta\rangle /\left(I_{e q} / a B\right)$ [51]. Again, we use the transformation between the rotational transform at $r / a=2 / 3$ and $I_{e q}$ given by equation (5) to get normalized beta and density values, which are assumed to be comparable to those in tokamaks. Most of the normalized data points of W7-AS and LHD are beyond the range of tokamaks. The strong correlation between $\beta_{N}$ and the Greenwald factor $n / n_{G W L}$ is mainly caused by the application of $I_{e q}$ for normalization in both directions.

\section{Discussion and Conclusions}

The documentation of experimental data and of parameters characterizing different experiment scenarios in different stellarators and helical devices is an important goal of the international stellarator database activity. Also, inter-machine comparisons and joint experiments are considered to be essential for developing the stellarator concept towards a fusion reactor. The inclusion of high beta data from W7-AS and LHD in the ISHCDB database provides an important test for the validity of existing scaling laws of 
the confinement in the high- $\beta$ regime and can lead to more reliable extrapolations to the reactor regime. In order to achieve this goal, results of local transport analyses have to be supplemented in the frame of the ISHPDB activity. This is currently in progress based on selected configurations in LHD [52] and W7AS. Detailed information about the mechanisms that limit the achievable $\beta$ and about their dependence on the magnetic configuration require advanced 3D equilibrium modeling (e.g. by HINT or PIES) allowing to assess effects of magnetic islands and stochastic field on the formation of self-consistent pressure profiles.

The first rough global confinement analysis presented in the previous chapters provides only limited and ambiguous information. In particular, the dependence of the global and local confinement on beta and the collisionality is not yet fully understood. The Bayesian approach of basic confinement model comparison is considered to be a powerful tool. This can help to make the scaling of the confinement more consistent with physics models and thus more reliable. Also, it can help to identify gaps in the parameter space of the collected data and thus provide some guidance for the planning of dedicated experiments.

The high- $\beta$ regimes in W7-AS and LHD are characterized by a parameter space which is close to operational limits depending on the configuration and on plasma parameters. In order to clarify the role of the configuration dependent beta limits imposed by confinement, equilibrium and stability effects, MHD related data are foreseen to be provided in the ISHPD and/or in a MHD-database, in addition to data required for local transport studies. Configuration parameters, data characterizing the MHD mode activity (e.g. onset parameters, saturation amplitudes, frequencies, mode numbers) and its relevance for the global and local transport, data on local pressure profiles and results of numerical equilibrium and stability calculations will be included.

An attempt was made to compare stellarator / heliotron data on global confinement and operational boundaries with those of tokamaks. The approach to formally convert parameters defined in tokamaks (e.g. plasma current) into equivalent parameters in currentless stellarators may not reflect the underlying confinement and stability physics correctly. Actually, the physics of the density limit and the $\beta$-limit seems to be quite different. In particular, the density limit in stellarators is of a radiative nature, which can be shifted up by increased heating power. The beta limit in stellarators appears to be not linked with MHD instabilities and manifests itself by a progressive deterioration of the confinement.

This work contributes to the International Stellarator Profile Data Base (ISHPDB) under auspices of the IEA Implementing Agreement for Cooperation in the Development of the Stellarator Concept. The first author would like to thank for his invitation by NIFS as Foreign Guest Scientist. 


\section{References}

[1] Grieger G and W7X-Team 1998 "The Wendelstein 7X Project" J. Plasma Fusion Res. SERIES 1 53-56

[2] Zarnstorff M C, Berry L A, Brooks A, Fredrickson E, Fu G-Y, Hirshman S, Hudson S, Ku L-P, Lazarus E, Mikkelsen D et al 2001 "Physics of the compact advanced stellarator NCSX" Plasma Phys. Control. Fusion 43 A237-A249

[3] Stroth U, Murakami M, Dory R A, Yamada H, Okamura S, Sano F and Obiki T 1996 "Energy confinement scaling from the international stellarator database" Nucl. Fusion 36 1063-1077

[4] Yamada H, Harris J H, Dinklage A, Ascasibar E, Sano F, Okamura S, Talmadge J, Stroth U, Kus A, Murakami S et al 2005 "Characterization of energy confinement in net-current free plasmas using the extended International Stellarator Database" Nucl. Fusion 45 1684-1693

[5] Watanabe K Y, Weller A, Sakakibara S, Narushima Y, Ohdachi S, Narihara K, Tanaka K, Ida K, Toi K, Yamada H et al 2004 "Progress of High-Beta Experiments in Stellarator/Heliotron" Fusion Sci. Technol. 46 24-33

[6] Weller A, Geiger J, Werner A, Zarnstorff M C, Nührenberg C, Sallander E, Baldzuhn J, Brakel R, Burhenn R, Dinklage A et al 2003 "Experiments close to the beta-limit in W7-AS" Plasma Phys. Control. Fusion 45 A285-A308

[7] Weller A, Sakakibara S, Watanabe K Y, Toi K, Geiger J, Zarnstorff M C, Hudson S R, Reiman A, Werner A, Nührenberg C et al 2006 "Significance of MHD Effects in Stellarator Confinement" Fusion Sci. Technol. 50 158-170

[8] Yamada H, Ida K, Murakami S, Watanabe K Y, Ascasibar E, Brakel R, Dinklage A, Harris J H, Okamura S, Sano F et al 2004 "Configuration effect on energy confinement and local transport in LHD and contribution to the International Stellarator Database" Fusion Sci. Technol. 46 82-90

[9] Hirsch M, Baldzuhn J, Beidler C, Brakel R, Burhenn R, Dinklage A, Ehmler H, Endler M, Erckmann V, Feng Y et al 2008 "Major results from the stellarator Wendelstein 7-AS" Plasma Phys. Contr. Fusion $\mathbf{5 0} 053001$

[10] Motojima O, Yamada H, Komori A, Ohyabu N, Mutoh T, Kaneko O, Kawahata K, Mito T, Ida $\mathrm{K}$, Imagawa S et al 2007 "Extended steady-state and high-beta regimes of net-current free heliotron plasmas in the Large Helical Device" Nucl. Fusion 47 S668-S676

[11] Sakakibara S, Watanabe K Y, Suzuki Y, Narushima Y, Ohdachi S, Nakajima N, Watanabe F, Garcia L, Weller A, Toi K et al 2008 "Study of Reactor-Relevant High-Beta Regime in the Large Helical Device" Plasma Phys. Contr. Fusion 50124014

[12] Ohdachi S, Watanabe K Y, Sakakibara S, Yamada H, Narushima Y, Funaba H, Suzuki Y, Toi K, Yamada I, Minami T et al "Two approaches to the reactor-relevant high-beta plasmas with profile control in the Large Helical Device" 22nd IAEA Fusion Energy Conf.

(Geneva,Switzerland) paper EX/8-1Rb 2009 Nucl. Fusion submitted

[13] Dinklage A, Ascasibar E, Beidler C D, Brakel R, Burhenn R, Castejon F, Estrada T, Funaba H, Feng Y, Geiger J et al 2007 "Status of the International Stellarator/Heliotron Profile Database" Proc. of ITC/ISHW2007 (CD) Toki, Japan (2007) P1-037 http://www.nifs.ac.jp/itc/itc17/file/PDF_proceedings/poster1/P1-037.pdf

[14] Funaba H, Watanabe K, Murakami S, Sakakibara S, Yamada H, Narihara K, Yamada I, Tanaka K, Tokuzawa T, Osakabe M et al 2008 "Configuration Effects on Local Transport in High Beta LHD Plasmas" Plasma and Fusion Research 3022

[15] Sakakibara S, Watanabe K Y, Yamada H, Narushima Y, Yamaguchi T, Toi K, Ohdachi S, Weller A, Tanaka K, Narihara K et al 2006 "Recent Progress of MHD Study in High-Beta Plasmas of LHD" Fusion Sci. Technol. 50 177-185

[16] Preuss R, Dinklage A, Weller A and W7-AS-team 2007 "Energy-Confinement Scaling for High- $\beta$ plasmas in the W7-AS Stellarator" Phys. Rev. Lett. 99245001

[17] Dinklage A, Maaßberg H, Preuss R, Turkin Y, Yamada H, Ascasibar E, Beidler C D, Funaba T, Harris J H, Kus A et al 2007 "Physical model assessment of the energy confinement time scaling in stellarators" Nucl. Fusion 47 1265-1273 
[18] Renner H, Anabitarte E, Ascasibar E, Besshou S, Brakel R, Burhenn R, Cattanei G, Dodhy A, Dorst D, Elsner A et al 1989 "Initial operation of the Wendelstein 7AS advanced stellarator" Plasma Phys. and Control. Fusion. 31 1579-1596

[19] Wagner F, Bäumel S, Baldzuhn J, Basse N, Brakel R, Burhenn R, Dinklage A, Dorst D, Ehmler H, Endler M et al 2005 "W7-AS: One step of the Wendelstein stellarator line" Phys. of Plasmas 12072509

[20] Grigull P, McCormick K, Baldzuhn J, Burhenn R, Brakel R, Ehmler H, Feng Y, Gadelmeier F, Giannone L, Hartmann D et al 2001 "First island divertor experiments on the W7-AS stellarator" Plasma Phys. Control. Fusion 43 A175-A193

[21] Iiyoshi A, Komori A, Ejiri A, Emoto M, Funaba H, Goto M, Ida K, Idei H, Inagaki S, Kado S et al 1999 "Overview of the Large Helical Device project" Nucl. Fusion 39 1245-1256

[22] Ohyabu N, Narushima Y, Nagayama Y, Narihara K, Morisaki T, Komori A and LHDExperimental-Group 2005 "Bifurcation of equilibria between with and without a large island in the large helical device" Plasma Phys. Control. Fusion 47 1431-1438

[23] Reiman A, Zarnstorff M C, Monticello D, Weller A, Geiger J and W7-AS-Team 2007 "Pressureinduced, Localized Breaking of Equilibrium Flux Surfaces in the W7AS Stellarator" Nucl. Fusion $47572-578$

[24] Suzuki Y, Nakajima N, Watanabe K, Nakamura Y and Hayashi T 2006 "Development and application of HINT2 to helical system plasmas" Nucl. Fusion 46 L19-L24

[25] Watanabe K Y, Suzuki Y, Yamaguchi T, Narihara K, Tanaka K, Tokuzawa T, Yamada I, Sakakibara S, Narushima Y, Morisaki T et al 2007 "Change of plasma boundaries due to beta in heliotron plasma with helical divertor configuration" Plasma Phys. Control. Fusion 49 605-618

[26] Hirshman S P, Rij W I v and Merkel P 1986 "Three-dimensional free boundary calculations using a spectral Green's function method" Comp. Phys. Commun. 43 143-155

[27] Spong D A, Hirshman S P, Berry L A, Lyon J F, Fowler R H, Strickler D J, Cole M J, Nelson B N, Williamson D E, Ware A S et al 2001 "Physics issues of compact drift optimized stellarators" 41 711-716

[28] Zarnstorff M C, Weller A, Fredrickson E, Knauer J P, Monticello D, Reiman A, Zegenhagen S, W7-AS-Team and NBI-Group 2005 "The Saturation of Beta in W7-AS" 32nd EPS Conference on Plasma Phys. Tarragona, Spain ECA Vol.29C P1.062

[29] Murakami M and al. e 1991 "Bootstrap current experiments in a toroidal plasma-confinement device" Murakami M, Carreras B A, Baylor L R, Bell G L, Bigelow T S, England A C, Glowienka J C, Howe H C, Jernigan T C, Lee D K et al 1991 "Bootstrap current experiments in a toroidal plasma-confinement device" Phys. Rev. Lett. 66707

[30] Funaba H, Watanabe K, Sakakibara S, Yamada I, Tanaka K, Tokuzawa T, Osakabe M, Narushima Y, Nakajima N, Yokoyama M et al 2007 "Transport Analysis of High-Beta Plasmas on LHD" Fusion Sci. Technol. 51 129-137

[31] Ohyabu N, Morisaki T, Masuzaki S, Sakamoto R, Kobayashi M, Miyazawa J, Shoji M, Komori A, Motojima O and LHD-Experimental-Group 2006 "Observation of Stable Superdense Core Plasmas in the Large Helical Device" Phys. Rev. Lett. 97055002

[32] Yamada H, Sakamoto R, Miyazawa J, Kobayashi M, Morisaki T, Masuzaki S, Ohdachi S, Goto M, Ida K, Sakakibara S et al 2007 "Characterization and operational regime of high density plasmas with internal diffusion barrier observed in the Large Helical Device" Plasma Phys. Control. Fusion 49 B487-B496

[33] Watanabe K Y, Sakakibara S, Narushima Y, Funaba H, Narihara K, Tanaka K, Yamaguchi T, Toi K, Ohdachi S, Kaneko O et al 2005 "Effects of global MHD instability on operational high-beta regime in LHD" Nucl. Fusion 45 1247-1254

[34] ITER 1999 "Chapter 2: Plasma confinement and transport" Nucl. Fusion 39 2175-2249

[35] Uckan N A and ITER-Physics-Group 1990 "ITER Physics Design Guidelines: 1989" ITER Documentation Series 10 IAEA Vienna

[36] ITER 1999 "Chapter 1: Overview and summary" Nucl. Fusion 39 2137-2174

[37] Connor J W and Taylor J B 1977 "Scaling laws for plasma confinement" Nucl. Fusion 17 10471055

[38] Connor J W 1988 "Invariance principles and plasma confinement" Plasma Phys. Control. Fusion 30 619-650 
[39] Miyazawa J, Yamada H, Murakami S, Funaba H, Peterson B J, Osakabe M, Tanaka K, Sakakibara S, Inagaki S and LHD-Experimental-Group 2005 "Temperature dependence of the thermal diffusivity in high-collisionality regimes in the large helical device" Plasma Phys. Control. Fusion 47 801-813

[40] Miyamoto K 1980 "Plasma Physics for Nuclear Fusion" MIT Press Cambridge, Massachusetts and London, England

[41] Shafranov V D 1983 " Magnetohydrodynamic theory of plasma equilibrium and stability in stellarators: Survey of results" Phys. Fluids 26 357-364

[42] Pustovitov V D 1994 "Fundamental Stellarator MHD Theory" Journal of plasma and fusion research 70 943-991

[43] Harafuji K, Hayashi $\mathrm{T}$ and Sato $\mathrm{T} 1989$ "Computational study of three-dimensional magnetohydrodynamic equilibria in toroidal helical systems" J. Comput. Phys. 81 169-192

[44] Reiman A and Greenside H 1986 "Calculation of three-dimensional MHD equilibria with islands and stochastic regions" Comp. Phys. Commun. 43 157-167

[45] Suzuki Y, Watanabe K Y, Sakakibara S, Nakajima N and Ohyabu N "Theoretical studies of equilibrium beta limit in heliotron plasmas" 22nd IAEA Fusion Energy Conference Geneva Switzerland paper TH/P9-19

[46] Weisen H, Alberti S, Berry S, Behn R and Blanchard P 1997 "Effect of plasma shape on confinement and MHD behaviour in TCV" Plasma Phys. Control. Fusion 39 B135-B144

[47] Sudo S, Takeiri Y, Zushi H, Sano F, Itoh K, Kondo K and Iiyoshi A 1990 "Scalings of Energy Confinement and Density Limit in Stellarator Heliotron Devices" Nucl. Fusion 30 11-21

[48] Giannone L, Baldzuhn J, Burhenn R, Grigull P, Stroth U, Wagner F, Brakel R, Fuchs C, Hartfuss $\mathrm{H} \mathrm{J}$, McCormick K et al 2000 "Physics of the density limit in the W7-AS stellarator" Plasma Phys. Contr. Fusion 42 603-627

[49] Giannone L, Burhenn R, McCormick K, Brakel R, Feng Y, Grigull P, Igitkhanov Y, NBI-Team, ECRH-Team and W7-AS-Team 2002 "Radiation power profiles and density limit with a divertor in the W7-AS stellarator" Plasma Phys. Contr. Fusion 44 2149-2165

[50] Greenwald M 2002 "Density limits in toroidal plasmas" Plasma Phys. Control. Fusion 44 R27R80

[51] Strait E J 1994 "Stability of high beta tokamak plasmas" Phys. Plasmas 1 1415-1431

[52] Funaba H, Dinklage A, H. Yamada, Yokoyama M, Watanabe K Y, Narihara K, Yamada I, Tanaka K, Tokuzawa T, Murakami S et al 2007 "Data Structure for LHD Plasmas in the International Stellarator/Heliotron Profile Database" Proc. of ITC/ISHW2007 (CD) Toki, Japan (2007) P1-040 http://www.nifs.ac.jp/itc/itc17/file/PDF_proceedings/poster1/P1-040.pdf 
Tables:

Table 1. Probabilities of model assessment for W7-AS and LHD high- $\beta$ survey subsets

\begin{tabular}{lcccc}
\hline CT-model & W7-AS & LHD, $\gamma=\mathbf{1 . 2 0}$ & $\begin{array}{c}\text { LHD, } \gamma=\mathbf{1 . 2 0} \\
(\boldsymbol{\beta}<\mathbf{3 . 5 \%})\end{array}$ & $\begin{array}{c}\text { LHD, } \gamma=\mathbf{1 . 2 0} \\
\left(\boldsymbol{v}^{*}>\mathbf{1}\right)\end{array}$ \\
\hline Collisionless low- $\beta$ & $10^{-52}$ & $10^{-129}$ & $10^{-40}$ & $10^{-100}$ \\
Collisional low- $\beta$ & $10^{-43}$ & $10^{-80}$ & $10^{-21}$ & $10^{-56}$ \\
Collisionless high- $\beta$ & $10^{-36}$ & $\mathbf{1}$ & $\mathbf{0 . 9 1}$ & $\mathbf{0 . 9 9 7}$ \\
Collisional high- $\beta$ & $\mathbf{1}$ & $10^{-4}$ & 0.09 & 0.003 \\
Ideal fluid & $10^{-45}$ & $10^{-98}$ & $10^{-21}$ & $10^{-45}$ \\
Resistive fluid & $10^{-41}$ & $10^{-18}$ & $10^{-16}$ & $10^{-35}$ \\
\hline
\end{tabular}


Figures:

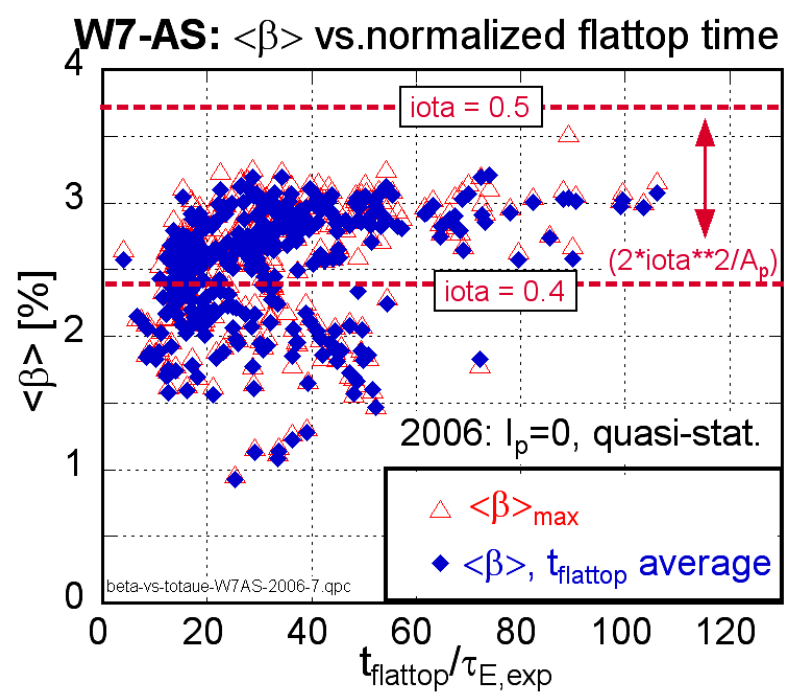

Figure 1. Values of $\langle\beta\rangle$ taken from the $2006 \mathrm{~W} 7$-AS survey dataset versus the time (normalized to $\tau_{E, \text { exp. }}$ ) in which $\delta\langle\beta\rangle /\langle\beta\rangle \leq 10 \%$ (as an index of the stationarity of the discharges). The horizontal dashed lines mark estimates of an equilibrium limit in a linearized low- $\beta$ approximation for two representative configurations of different rotational transform in the W7-AS device, taking the reduction of the Shafranov shift by a factor of about two (resulting from stellarator optimization in W7-AS) into account. 


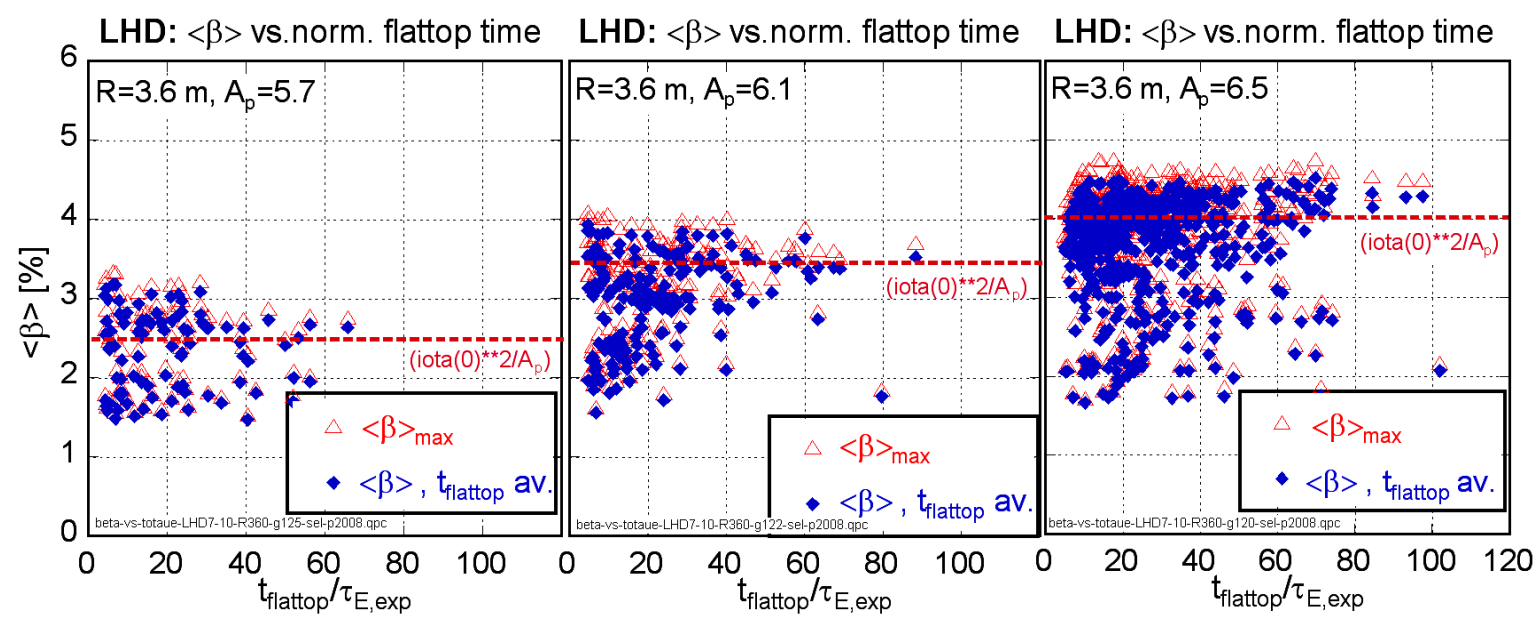

Figure 2. $\langle\beta\rangle$ values taken from the three new LHD survey datasets with $R_{a x}=3.6 \mathrm{~m}, \gamma=1.25,1.22,1.20$ (corresponding to aspect ratios of $A_{p}=5.7,6.1,6.5$ of the vacuum configurations) versus the time (normalized to $\tau_{E, \text { exp. }}$ ) in which $\delta\langle\beta\rangle /\langle\beta\rangle \leq 10 \%$. The horizontal dashed lines represent crude estimates of equilibrium limits in a low-shear stellarator (linear low- $\beta$ approximation), which underestimate significantly the predicted equilibrium limits of the high-shear LHD configurations. 


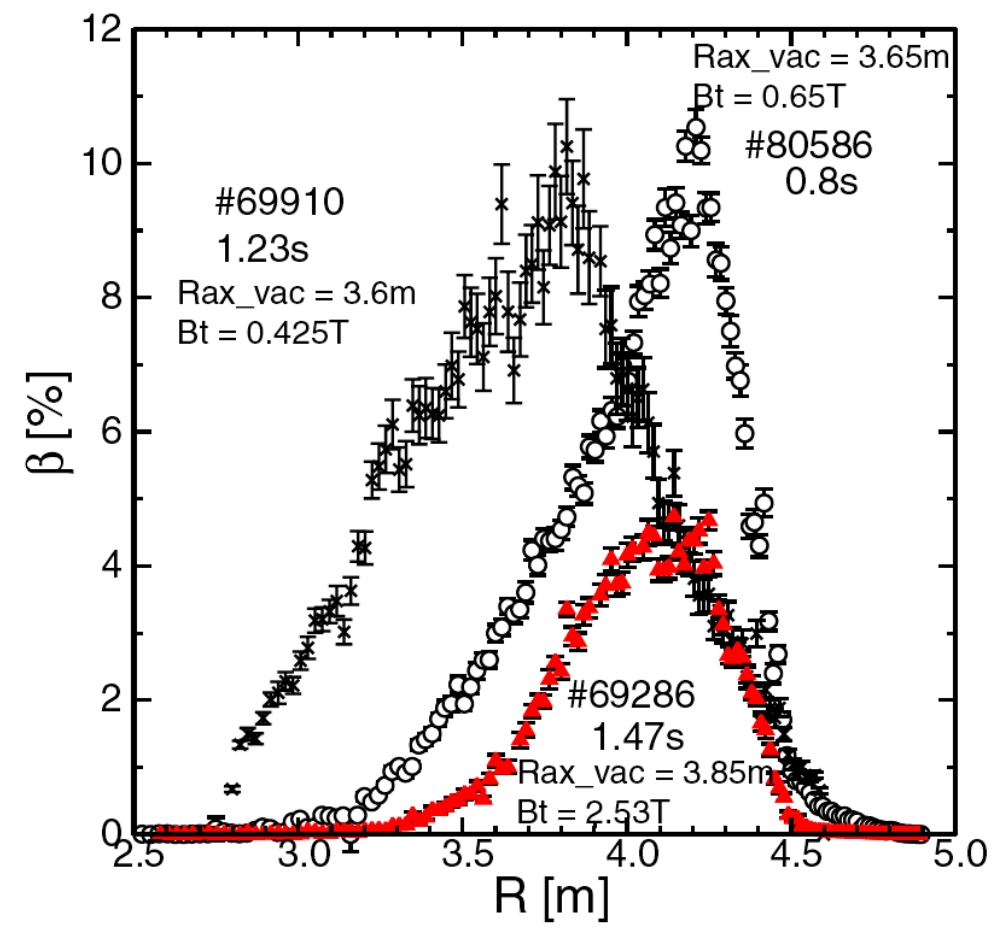

Figure 3. Achievement of high central $\beta$ in LHD in plasmas with internal diffusion barrier (IDB) generated by pellet fuelling in configurations with vacuum axis located further outward compared with the conventional high- $\beta$ configuration. The peaking factors $\beta_{\max } /\langle\beta\rangle$ of the pressure profiles of the IDB cases $(\# 80586, \# 69286)$ are clearly larger compared with the conventional, stationary high- $\beta$ inward shifted case (\#69910), in which $\beta_{\max } /\langle\beta\rangle \approx 2$ and $\langle\beta\rangle \approx 4.8 \%$ was achieved. 


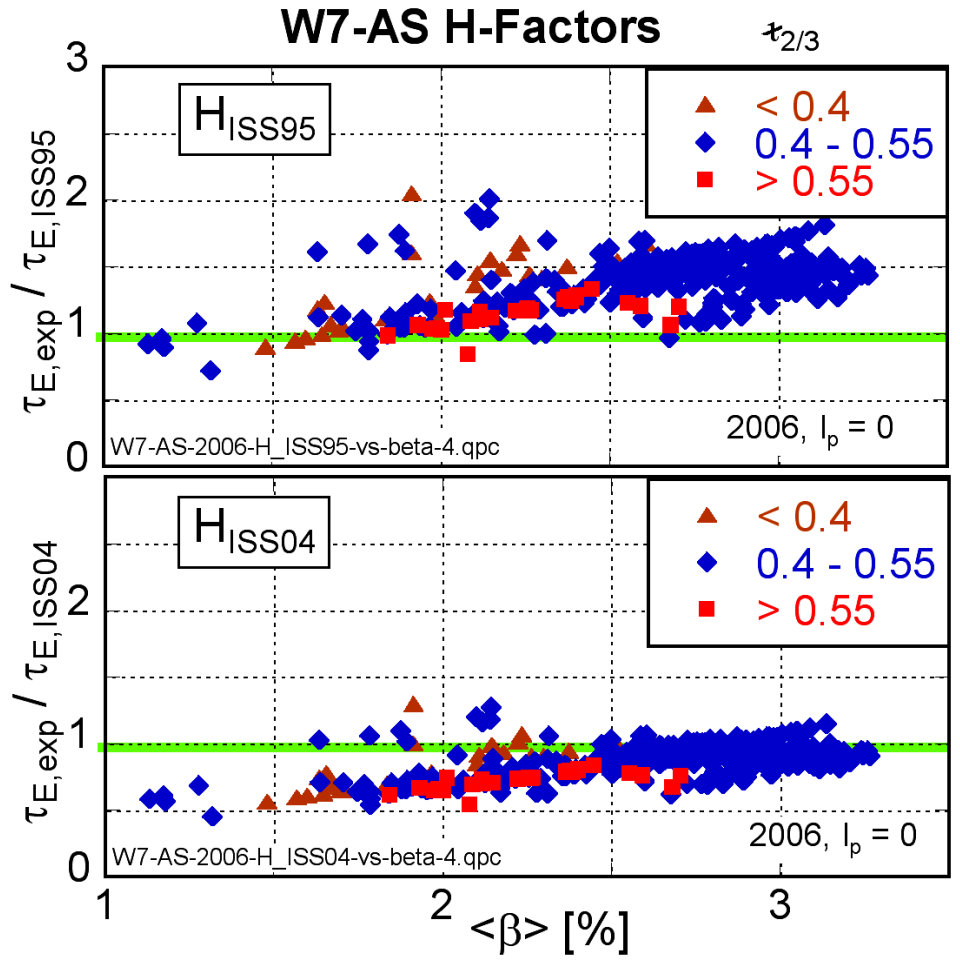

Figure 4. Energy confinement times normalized to ISS95 (top) and ISS04 (bottom). The data (2006 W7-AS dataset) refer to quasi-stationary, currentless discharges in various different configurations. The ISS04 renormalization factor ([4]) determined for these selected data yields $f_{\text {ren }}=0.86 \pm 0.18$. 


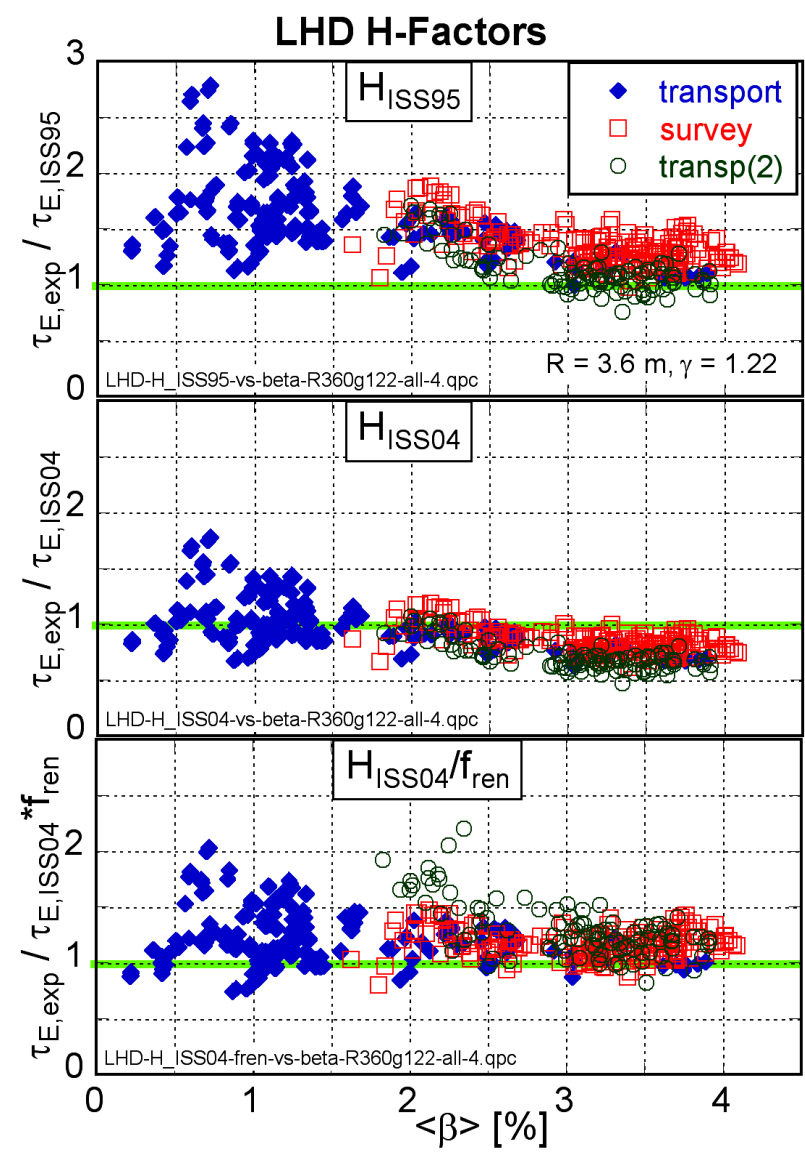

Figure 5. Energy confinement times normalized to ISS95 ( $\mathrm{H}_{\mathrm{ISS} 95}$, top) and ISS04 $\left(\mathrm{H}_{\mathrm{ISS} 04}\right.$, middle) for the LHD $\gamma=1.22$ configuration subset (3 different datasets, which yield an renormalization factor of $f_{\text {ren }}=0.87 \pm 0.21$ ). If the renormalization factors corresponding to the finite- $\beta$ axis position are applied (bottom), the confinement dependence on $\beta$ is much weaker. The transport data are arranged in two datasets (transport, $\operatorname{transp}(2)$ ), but the same analysis approach was used in the two cases. 


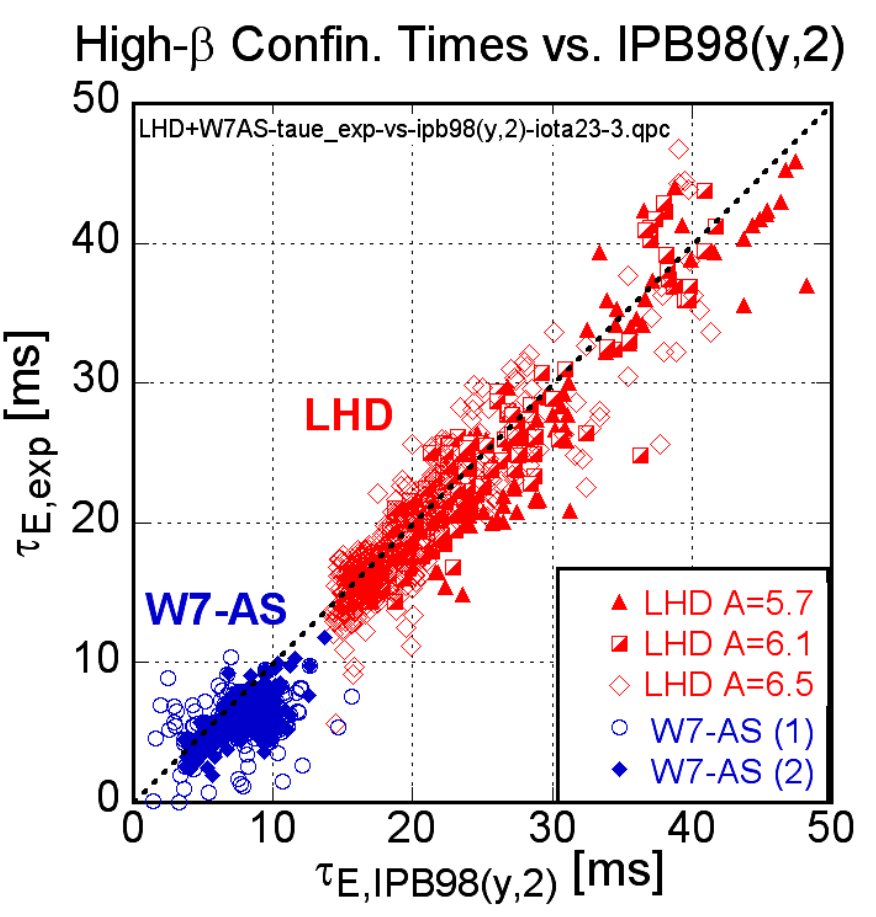

Figure 6. Measured energy confinement times compared with tokamak ELMy H-Mode scaling IPB98 $(\mathrm{y}, 2)$ for W7-AS and LHD high- $\beta$ survey datasets. In the scaling, corresponding toroidal currents were used which produce equivalent values of the stellarator rotational transform at $r / a=2 / 3$. 


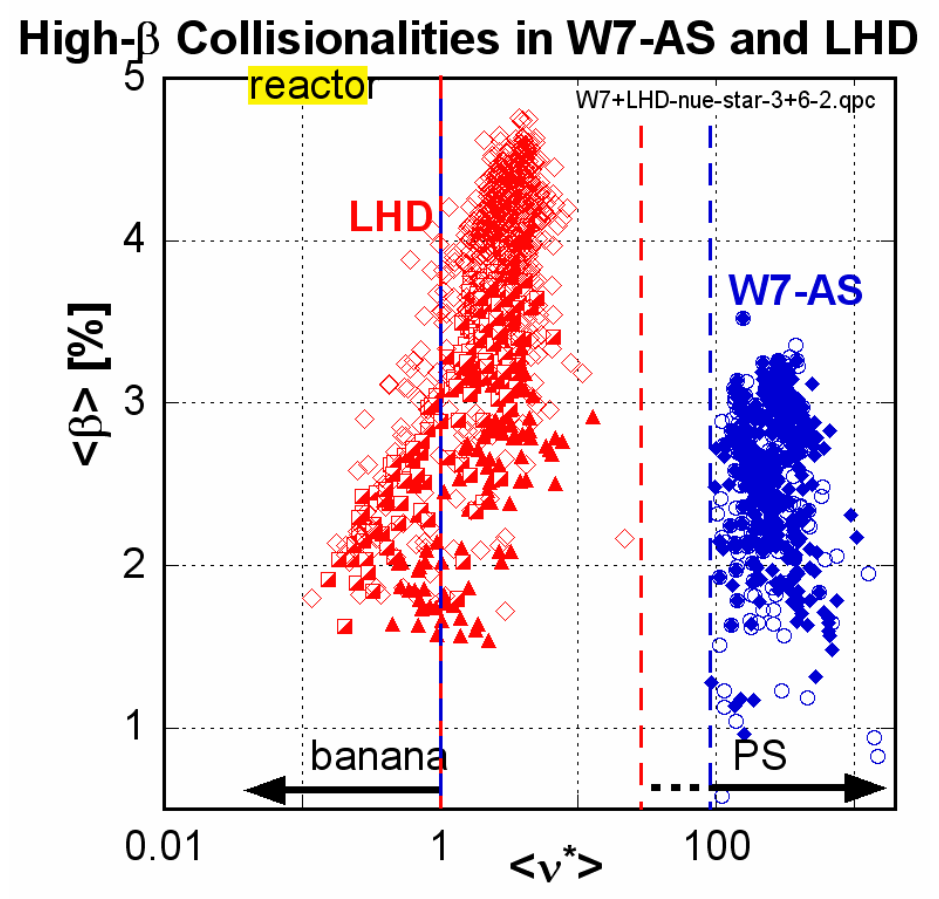

Figure 7. The high- $\beta$ plasmas of LHD and W7-AS are clearly in different collisionality regimes (W7-AS and LHD high- $\beta$ survey datasets, refer to legend in figure 6). The volume averaged collisionalities in W7-AS are entirely in the Pfirsch-Schlüter regime. In LHD, some co-linearity between beta and nue-star is indicated. 


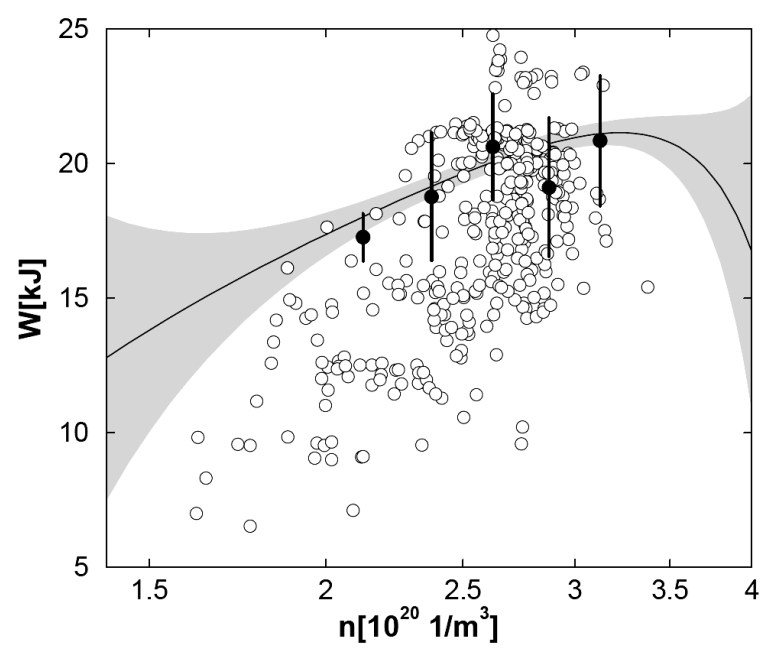

Figure 8. Scaling of the global confinement derived for a high- $\beta$ data subset of W7-AS (open symbols) using constraints imposed by comparison with a collisional high- $\beta$ Connor-Taylor model. The dedicated experimental density-scan (solid symbols) agrees well with the predicted curve (solid line), which was obtained from all data points with open symbols. The shaded area marks a confidence range according to the Bayesian probability approach used in the analysis. 


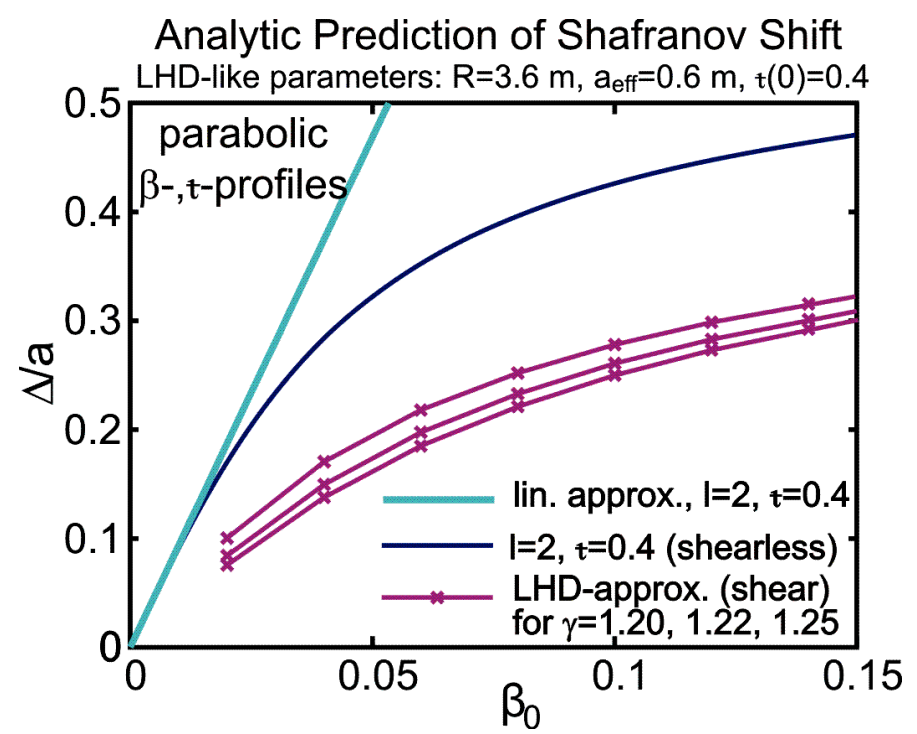

Figure 9. Extended analytic model for the Shafranov shift using basic parameters of LHD inward shifted high- $\beta$ configurations. The linear $\beta$-dependence (low- $\beta$ approximation) turns into a square root dependence. In the centre $t=0.4$ is used in all cases. The Shafranov shift is significantly reduced in the presence of LHD-like iota-profiles with edge values of $t(a) \approx 1.6$ and stays below $\Delta / a \approx 0.3$ up to the highest $\beta$-values achieved in LHD. 


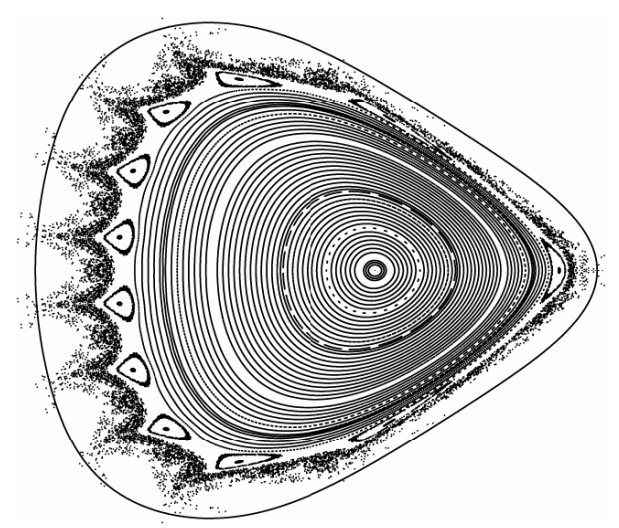

Figure 10. Beta induced formation of a stochastic field layer in a W7-AS finite- $\beta$ equilibrium $(\langle\beta\rangle=1.9 \%)$ as reconstructed with the PIES code. The vacuum configuration has a flat rotational transform around $\boldsymbol{t}_{v a c} \approx 0.45$. Plasma pressure is sustained up to the plasma boundary as determined by the STELLOPT code (outermost contour). 


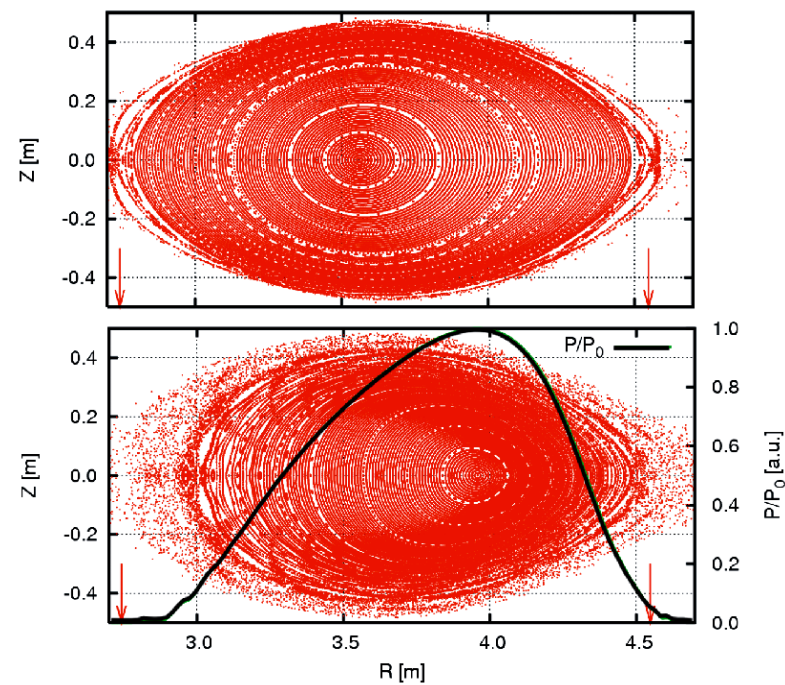

Figure 11. Modification of the magnetic field structure by finite- $\beta$ effects in LHD modelled by the HINT code. For comparison, the vacuum configuration is shown on the top. The lower part contains an equilibrium corresponding to the maximum beta achieved in the $R_{a x}=3.6 \mathrm{~m}, \gamma=1.25$ configuration, where $\langle\beta\rangle \sim 3 \%$. Plasma pressure (overlaid line) is sustained across the stochastic field region at the plasma boundary. 


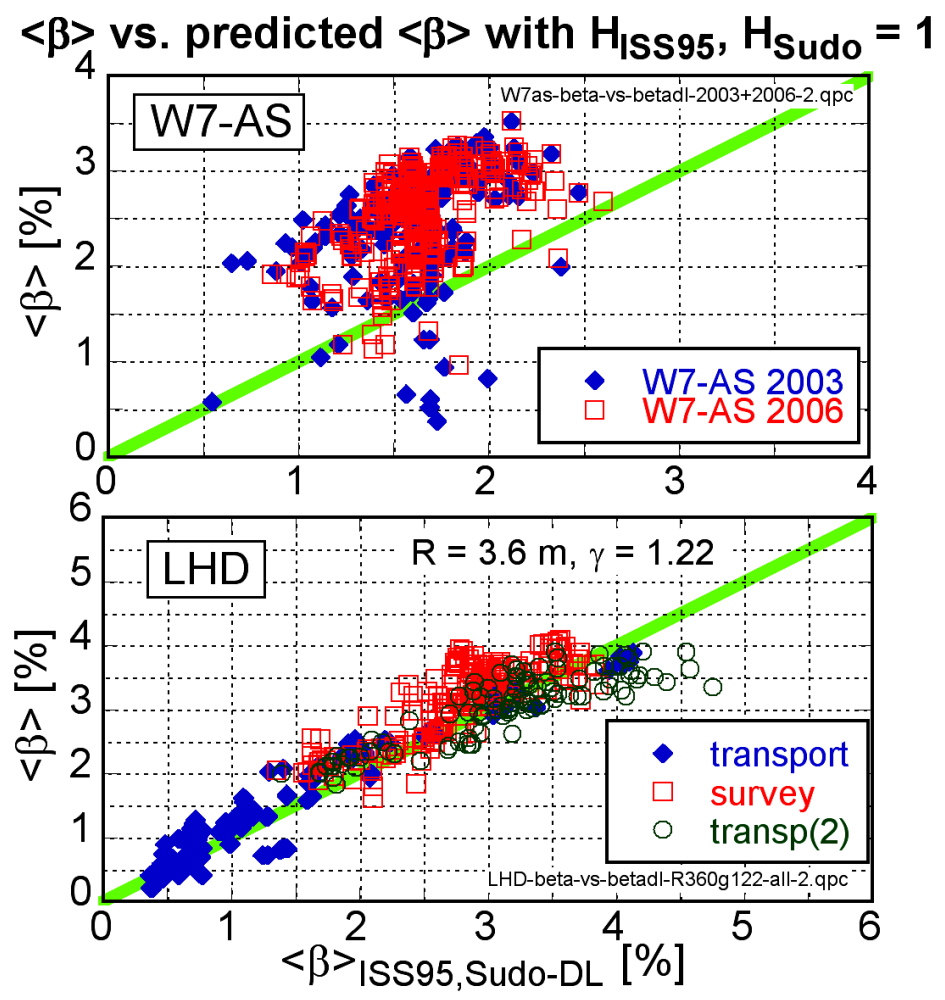

Figure 12. Predicted confinement limit of $\langle\beta\rangle$ compared with experimental values, assuming $H_{I S S 95}=1$ and densities at the Sudo-limit in the ISS95 scaling. 

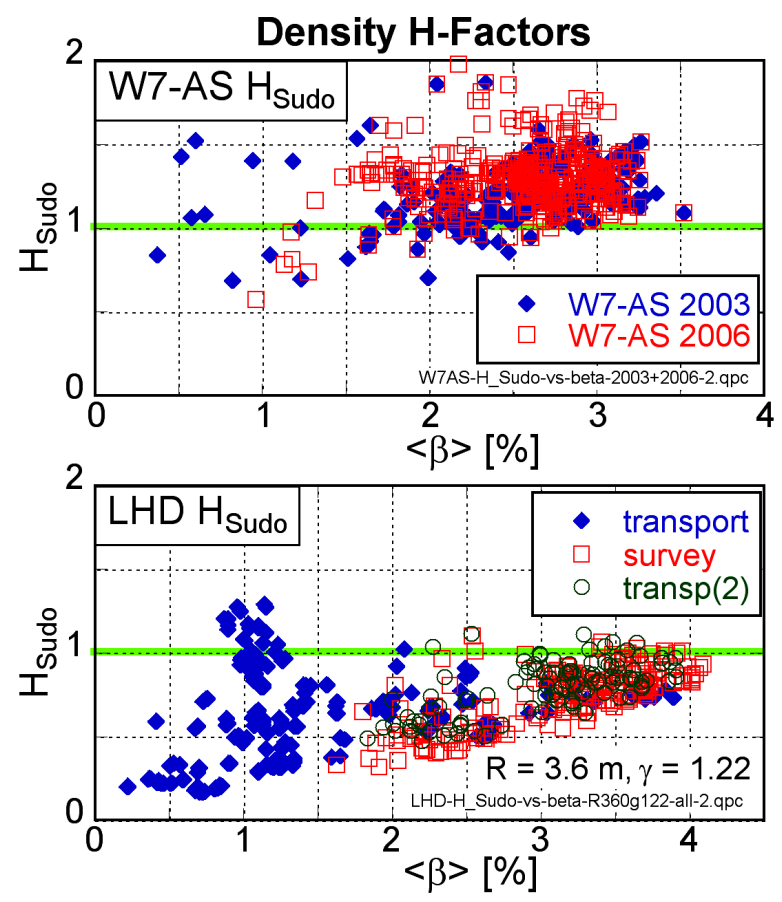

Figure 13. Densities in the high- $\beta$ regime normalized to the Sudo density limit. Top: in W7-AS densities exceed the Sudo limit, volume averaged densities of up to $2.5 \cdot 10^{20} \mathrm{~m}^{-3}$ are reached. Bottom: results for the configuration with $R_{a x}=3.6 \mathrm{~m}, \gamma=1.22$ and for 3 data subsets (details described in caption of figure 5). At high beta a co-linearity between density and beta exists. 


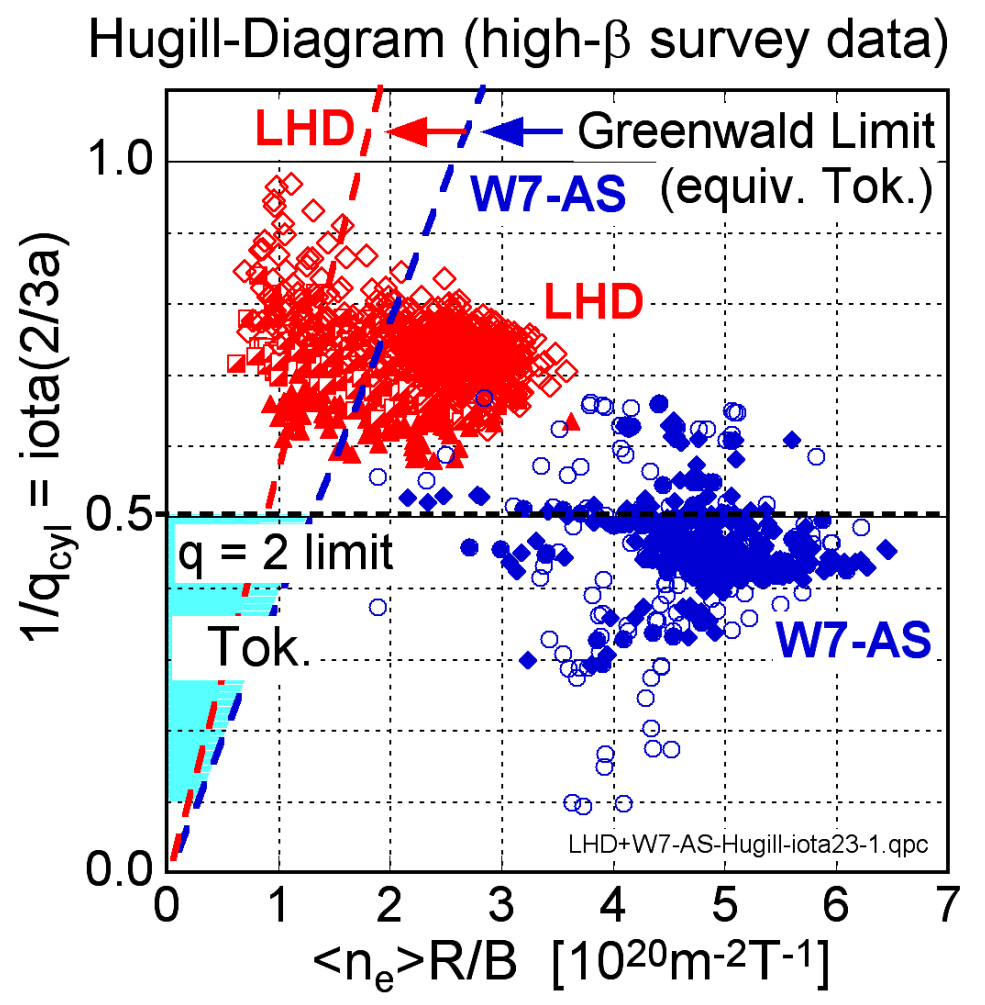

Figure 14. Normalized densities of the LHD and W7-AS high- $\beta$ survey data subsets in the tokamak Hugill diagram representation, where $1 / q_{c y l}$ (measure of the tokamak current) was replaced by the values of the stellarator rotational transform at $r / a=2 / 3$. The dashed lines represent Greenwald limits estimated for LHD and W7-AS using an equivalent current reproducing the rotational transform at $r / a=2 / 3$. The symbols are the same as in figure 15 and are explained there. 


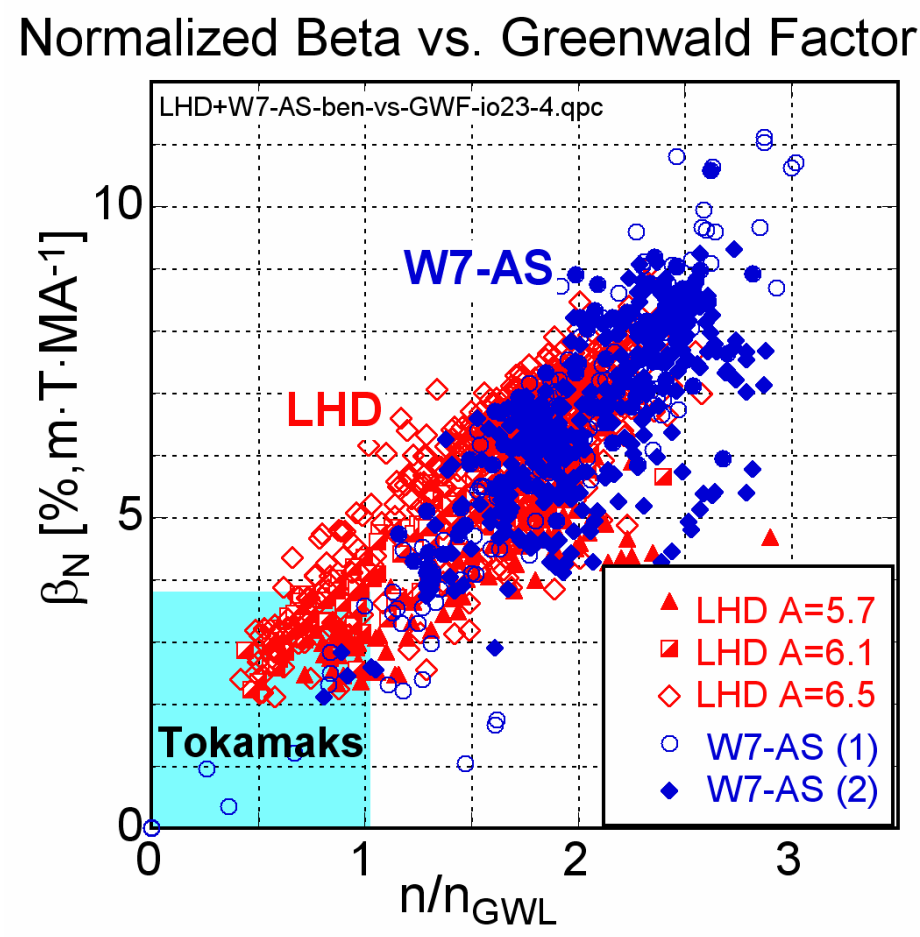

Figure 15. LHD and W7-AS high- $\beta$ survey data projected into a tokamak operational diagram spanned by the normalized $\beta$ and the density normalized to the Greenwald limit. The stellarator rotational transform at $r / a=2 / 3$ was used for the assessment of an equivalent tokamak current that produces about the same volume averaged rotational transform. The different symbols mark configurations in LHD differing by their aspect ratio $A$ (or $\gamma$-parameter). For W7-AS, the 2003 (1) and the 2006 (2) datasets are distinguished. 